\title{
An integrated perfusion machine preserves injured human livers for 1 week
}

\author{
Dilmurodjon Eshmuminov ${ }^{1,2,6}$, Dustin Becker ${ }^{2,3,6}$, Lucia Bautista Borrego ${ }^{1,2}$, Max Hefti ${ }^{2,3}$, \\ Martin J. Schuler ${ }^{2,3}$, Catherine Hagedorn ${ }^{1,2}$, Xavier Muller ${ }^{1,2}$, Matteo Mueller ${ }^{1,2}$, Christopher Onder ${ }^{2,4}$, \\ Rolf Graf ${ }^{1,2}$, Achim Weber $\mathbb{1}^{5}$, Philipp Dutkowski ${ }^{1,2}$, Philipp Rudolf von Rohr ${ }^{2,3,7}$ and \\ Pierre-Alain Clavien ${ }^{10,2,7 \star}$
}

The ability to preserve metabolically active livers ex vivo for 1 week or more could allow repair of poor-quality livers that would otherwise be declined for transplantation. Current approaches for normothermic perfusion can preserve human livers for only $24 \mathrm{~h}$. Here we report a liver perfusion machine that integrates multiple core physiological functions, including automated management of glucose levels and oxygenation, waste-product removal and hematocrit control. We developed the machine in a stepwise fashion using pig livers. Study of multiple ex vivo parameters and early phase reperfusion in vivo demonstrated the viability of pig livers perfused for 1 week without the need for additional blood products or perfusate exchange. We tested the approach on ten injured human livers that had been declined for transplantation by all European centers. After a 7-d perfusion, six of the human livers showed preserved function as indicated by bile production, synthesis of coagulation factors, maintained cellular energy (ATP) and intact liver structure.

S tandard preservation of donor livers is performed by flushing the organ with a cold solution at the time of procurement, which is followed by static storage on ice. This approach reduces liver metabolic activity, allowing safe storage for up to $12-18 \mathrm{~h}$ (refs. ${ }^{1,2}$ ). Recently, the combination of supercooling with subzero storage $\left(-4^{\circ} \mathrm{C}\right)$ and hypothermic, as well as subnormothermic, machine perfusion achieved an extension of the preservation time of human livers up to $27 \mathrm{~h}$ (ref. ${ }^{2}$ ). By contrast, human livers can also be kept metabolically fully active for up to $24 \mathrm{~h}$ by supplying normothermic blood or oxygenated fluids in a controlled environment by machine perfusion $^{1,3-5}$. The possibility of repairing poor-quality livers sufficiently to enable transplantation requires preserving metabolically active livers ${ }^{1,3}$ for several days. Accordingly, the need for long-term organ preservation technology has been endorsed by multiple private and governmental initiatives ${ }^{1}$. However, currently used normothermic machine perfusion technologies have been used only for a relatively short time period (for example, a median perfusion time of $9 \mathrm{~h}\left(\right.$ ref. $\left.^{5}\right)$ ), to successfully maintain hemodynamics, perfusate oxygenation and temperature.

We sought to extend perfusion time beyond $24 \mathrm{~h}$ by engineering a perfusion machine that recapitulates additional core body functions that are critical to liver health. We chose 7-d as a target because this time period has been shown to offer a credible time frame for inducing clinically relevant liver regeneration in patients undergoing complex liver resection ${ }^{6-8}$. Under physiologic conditions, the liver, which constitutes $2.5 \%$ of body weight, receives $25 \%$ of the blood output of the heart and performs $>5,000$ functions ${ }^{9}$. It has a unique dual vascular supply with high-pressure, oxygen-rich arterial blood entering through the hepatic artery and low-pressure, oxygen-reduced portal vein blood draining the abdominal viscera. Its high metabolic activity produces waste products that are excreted in the bile or removed by hepatic macrophages or the kidneys. The metabolic profile is largely controlled by pancreatic hormones, including insulin and glucagon. Our perfusion technology, developed in the "Liver4Life project", includes automated control of glucose levels by injection of insulin and glucagon, a dialysis membrane for waste-product removal, regulation of oxygenation and liver movement to prevent pressure necrosis.

\section{Results}

Liver perfusion machine. Our perfusion machine (Fig. 1a,b) recapitulates blood supply through the two vascular entries of the liver, the hepatic artery and portal vein. The hepatic artery is supplied with oxygen-rich blood at elevated pressure (mean arterial pressure $(\mathrm{MAP}) \geq 65 \mathrm{mmHg}$ ) in a pulsatile manner (Fig. 1c), whereas the portal vein receives blood at low pressure (around 5-10 $\mathrm{mmHg}$ ) with a reduced oxygen content (venous blood, non-pulsatile). The system maintains oxygen saturation of $65 \%$ in the vena cava by continuously adjusting oxygen content in the portal vein (Fig. 1d). In vivo, nutrients and bile salts are transported from the intestine to the liver via the portal vein. To recapitulate this, we inject parenteral nutrition and ursodeoxycholic acid into the portal vein line of our perfusion machine. Hepatic artery hemodynamics are tightly controlled by automated infusion of vasoconstrictors and vasodilators (Fig. 1e) into the hepatic artery line. The pressure in the vena cava is continuously kept at physiological levels close to $0 \mathrm{mmHg}(0-2 \mathrm{mmHg})$ to prevent liver congestion. An oxygenator provides oxygen to the hepatic artery and to the portal vein while removing $\mathrm{CO}_{2}$ from

\footnotetext{
'Department of Surgery and Transplantation, Swiss Hepato-Pancreato-Biliary (HPB) Center, University Hospital Zurich, Zurich, Switzerland. ${ }^{2}$ Wyss Zurich, ETH Zurich and University of Zurich, Zurich, Switzerland. ${ }^{3}$ Transport Processes and Reactions Laboratory, Department of Mechanical and Process Engineering, ETH Zurich, Zurich, Switzerland. ${ }^{4}$ Institute for Dynamic Systems and Control, Department of Mechanical and Process Engineering, ETH Zurich, Zurich, Switzerland. ${ }^{5}$ Department of Pathology and Molecular Pathology, and Institute of Molecular Cancer Research (IMCR), University Zurich and University Hospital Zurich, Zurich, Switzerland. ${ }^{6}$ These authors contributed equally: Dilmurodjon Eshmuminov, Dustin Becker.

${ }^{7}$ These authors jointly supervised this work: Philipp Rudolf von Rohr, Pierre-Alain Clavien. *e-mail: clavien@access.uzh.ch
} 
the perfusate. The oxygen tension in the hepatic artery is continuously monitored and controlled to remain in predefined bounds, that is, $10-12 \mathrm{kPa}$. The $\mathrm{CO}_{2}$ tension is adapted via the oxygenator to maintain blood $\mathrm{pH}$ between 7.25 and 7.45 (Supplementary Fig. 1). We incorporated an integrated dialysis unit for physiologic electrolyte balance and removal of metabolic waste products from the blood.

We used an algorithm to automatically adjust the dialysate flow, controlling the concentration of red blood cells (hematocrit) on the basis of continuous measurements (Fig. 1f). Automated insulin (Fig. 1g) and glucagon administration was used to maintain physiological blood glucose levels (targeted range of 3.5-6.5 $\mathrm{mmoll}^{-1}$ ) in response to continuous glucose measurements. Continuous movement of the liver, in an effort to mimic diaphragm oscillations, is also integrated into the system (Supplementary Fig. 1). The perfusion machine is fully automated obviating the need for constant presence of personnel. Features of the described long-term perfusion technology as compared to the commercially available perfusion machines are summarized in Fig. $1 \mathrm{~h}$.

Through early experiments using pig livers we identified five major hurdles, each limiting successful long-term liver preservation with blood-based perfusate, namely, control of glucose metabolism, prevention of hemolysis, removal of waste products, control of perfusate oxygenation and simulation of diaphragm movement (Fig. 2). These obstacles were studied and overcome through incremental steps using 70 healthy pig livers, before initiating the human study.

Glucose metabolism. We first perfused pig livers with a hyperglycemic perfusate (perfusate glucose $>10 \mathrm{mmoll}^{-1}$ ) at fixed insulin injection rates $\left(0.9 \mathrm{U} \mathrm{h}^{-1}\right)$. Although glucose is not the primary energy source for hepatocytes ${ }^{10}$, perfusion protocols available in the literature ${ }^{4,5,11,12}$ have used this approach. For example, the pioneering randomized controlled trial of normothermic liver perfusion in humans also reported high glucose levels in the perfusate $\left(>10 \mathrm{mmoll}^{-1}\right)$ at a constant insulin injection rate $\left(7 \mathrm{Uh}^{-1}\right)^{5}$. However, using this approach, we observed high insulin concentrations in the perfusate $\left(229 \pm 72 \mathrm{pmoll}^{-1}\right)$, which led to activation of glycogen synthase and excessive glycogen deposition in liver tissue, similar to what is seen in glycogen-storage diseases (Fig. 2a,c and Supplementary Fig. 2).

In the next series of experiments, we reduced glucose levels in the perfusate to $4 \mathrm{mmoll}^{-1}$ by manually adjusting the glucose infusion rate resulting in considerable reduction of glycogen deposition (Fig. 2a). The persistence of high levels of insulin $\left(0.9 \mathrm{U} \mathrm{h}^{-1}\right)$, however, resulted in activation of glucose uptake by hepatocytes and deprivation of glucose for erythrocytes in the perfusate (erythrocytes rely only on glucose as an energy source owing to their lack of mitochondria), implying failure of manual control (Fig. $2 \mathrm{~b}$ and Supplementary Fig. 2). Therefore, we included a device with automated monitoring of glucose levels in the perfusate using a continuous online sensor (Figs. $1 \mathrm{~g}$ and 2a,c, and Supplementary Fig. 2). We implemented closed-loop control of glucose levels by automatically injecting insulin and glucagon (Supplementary Fig. 1).
Minimizing hemolysis. Hemolysis is a common shortcoming in liver perfusion systems using blood-based perfusates owing to exposure to foreign surfaces and non-pulsatile perfusion of the hepatic artery. Elevated hemolysis with a non-pulsatile pump is potentially explained by sludge and erythrocyte stasis, which is prevented with pulsatile perfusion ${ }^{13-16}$.

To decrease hemolysis in our system, we opted for a perfusion loop design with only one pump head and one oxygenator despite having a dual perfusion route. Pulsatile flow in the hepatic artery was generated by varying the rotational speed of the centrifugal pump head with a targeted MAP of $65 \mathrm{mmHg}$ and 60 beats per minute creating a pulse waveform that mimicked the in vivo setting (Fig. 1c). Pulsatile flow caused a significantly lower degree of hemolysis $\left(0.2 \pm 0.1 \mathrm{gl}^{-1}\right.$ versus $\left.0.7 \pm 0.2 \mathrm{gl}^{-1} ; P<0.001\right)$ after $24 \mathrm{~h}$ of perfusion (Fig. 2d) as compared to non-pulsatile flow.

Electrolyte control. We observed elevated sodium levels (hypernatremia) during liver perfusion, reaching levels of up to $200 \mathrm{mmoll}^{-1}$, which has detrimental effects on organ viability. Serum sodium concentrations of $>165 \mathrm{mmoll}^{-1}$ in liver donors is considered an extended criterion, which was observed in a human liver perfusion without an integrated dialysis system ${ }^{5,17}$. To prevent hypernatremia, we integrated a dialysis membrane in the perfusion loop, performing critical excretory kidney functions (Figs. 1f and 2e). An additional effect of dialysis was the automated modulation of a transmembrane flow (TMF) of dialysate across the dialysis filter membrane, which allows the control of the hematocrit level (Fig. 2f). These refinements enabled us to run perfusion for 7-d without the need for exchanging the perfusate or using any additional blood products. In addition, dialysis also removed metabolic waste products, such as blood urea nitrogen (BUN; Fig. 2g).

Physiologic venous oxygen saturation in the portal vein. Despite the uniform delivery of highly oxygenized blood through the hepatic artery, most reported liver perfusion systems provide non-physiologic arterial blood also through the portal vein ${ }^{4,5,11,12}$. However, in our preliminary experiments we found that hyperoxygenation in the portal vein was associated with the need for high amounts of vasodilators ( $285 \pm 162$ milliliter per week) to maintain a physiologic flow in the hepatic artery (Fig. 2h,i); a finding consistent with other reports indicating enhanced arterial vasoconstriction owing to hyperoxygenation ${ }^{18,19}$. To avoid over oxygenation in the portal vein, we mixed the deoxygenated venous blood from the blood reservoir with the freshly oxygenated blood from the oxygenator. An algorithm enabled automatic adjustment of oxygen saturation in the portal vein to maintain a target saturation of $65 \%$ in the vena cava (Fig. 1d). With this approach, the need for vasodilators decreased from $285 \pm 162$ milliliters per week to $41 \pm 34$ milliliters per week $(P=0.011)$, despite maintaining physiologic flow in the hepatic artery (Fig. 2i).

Liver movement. In the human body, the liver and other organs move rhythmically owing to attachment to the diaphragm. A consistent

Fig. 1 | Long-term liver perfusion machine specifications. a, Simplified schematics of the perfusion machine with all main components included. $\mathbf{b}$, Visual illustration of the perfusion loop. c, Representative illustration of hepatic-artery (HA) pressure pulse with the visible dicrotic notch (imitating in vivo aortic valve closure, red circle) created by variation of the rotation speed of the centrifugal pump. $\mathbf{d}$, Representative bypass flow of fully oxygenated (arterial) perfusate to the portal vein (PV), automatically controlled according to the monitored oxygen saturation of the deoxygenized perfusate (vena cava (VC)) to generate a physiological portal vein oxygen saturation (bypass seen in a). e, Representative illustration of hepatic-artery flow variation in relation to the injection rate of vasodilator (Flolan (Epoprostenol)) when the limit of $0.251 \mathrm{~min}^{-1}$ is undershot. $\mathbf{f}$, Representative illustration of the hematocrit control by dialysis, where the system continuously measures the hematocrit level in the perfusate and determines the volume of dialysis fluid that is administered into, or removed from the perfusion loop through the dialysis filter. $\mathbf{g}$, Representative illustration of the automated insulin injection shown for a $25 \mathrm{~h}$ section of perfusion where insulin is infused when the defined glucose target level is surpassed in the perfusate. $\mathbf{h}$, Illustrative table showing features of the presented long-term perfusion machine as compared to the commercially available perfusion machines. 'Liver4Life' refers to the name of the research group. 
observation of ex vivo livers perfused on static systems support is heterogeneous perfusion of the hepatic parenchyma and consecutive local pressure necrosis ${ }^{11,20,21}$ (Fig. 2j). In pilot studies, keeping the liver in situ in cadaveric pigs, we tested the importance of diaphragm movement by artificially maintaining lung ventilation. We found that ventilation-associated diaphragm movement prevented pressure necrosis (Fig. 2j). To simulate this movement in the ex vivo system, we placed the liver on a silicone mat, which was a

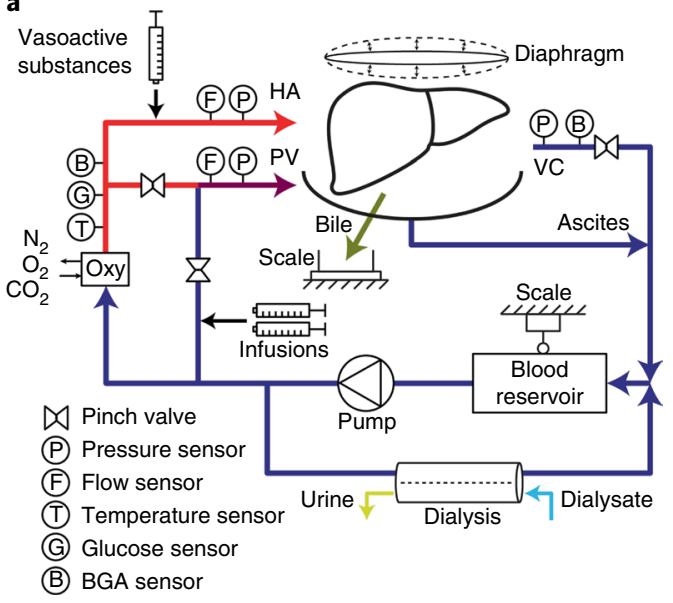

b

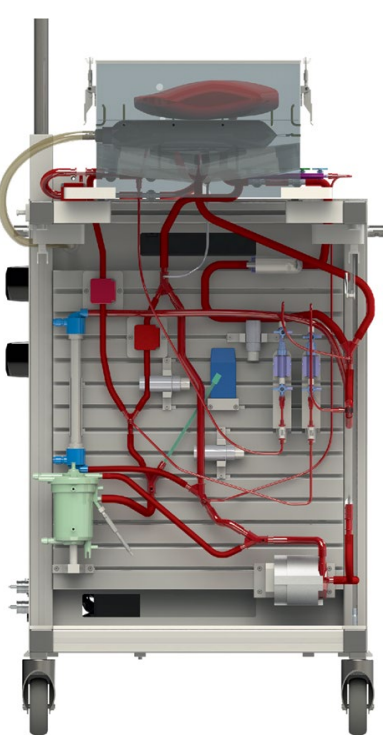

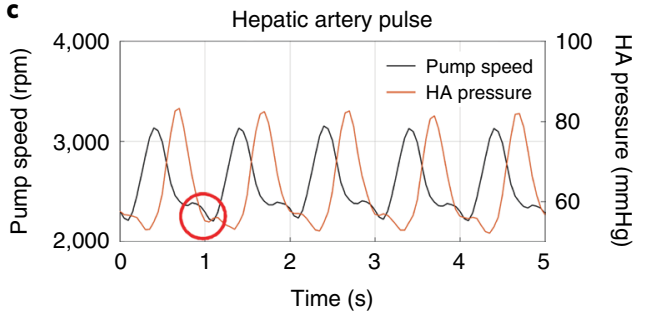

d

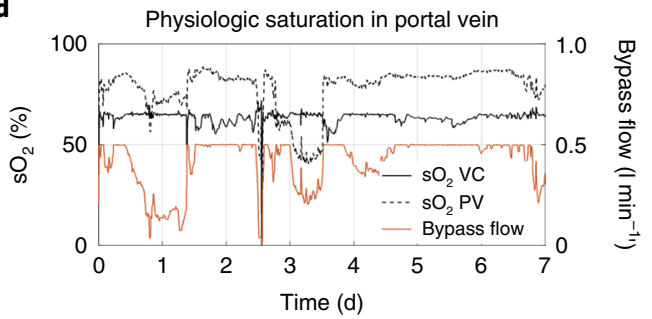

h

\begin{tabular}{|c|c|c|c|}
\hline \multirow{2}{*}{ Liver chamber } & \multirow{2}{*}{$\begin{array}{l}\text { Commercial } \\
\text { perfusion } \\
\text { machines } \\
\text { X }\end{array}$} & \multicolumn{2}{|c|}{$\begin{array}{l}\text { Long term perfusion machine } \\
\text { LEL }\end{array}$} \\
\hline & & $x$ & Safe liver storage \\
\hline $\begin{array}{l}\text { Pump } \\
\text { Continuous flow in PV } \\
\text { Pulsatile flow in HA }\end{array}$ & $\begin{array}{l}x \\
x\end{array}$ & $\begin{array}{l}x \\
x\end{array}$ & $\begin{array}{l}\text { Wave and pulse shape of flow } \\
\text { and pressure of commercial } \\
\text { machines not reported }\end{array}$ \\
\hline $\begin{array}{l}\text { Pressure/flow sensors } \\
\text { HA } \\
\text { PV } \\
\text { Vena cava }\end{array}$ & $\begin{array}{l}X \\
X \\
X\end{array}$ & $\begin{array}{l}x \\
x \\
x\end{array}$ & $\begin{array}{l}\text { Continuous pressure and flow } \\
\text { monitoring }\end{array}$ \\
\hline $\begin{array}{l}\text { Oxygenator } \\
\text { Gas supply } \\
\text { Heat exchange } \\
\text { Individual gas-supply } \\
\text { for pH control in blood }\end{array}$ & $\begin{array}{l}X \\
X\end{array}$ & $\begin{array}{l}X \\
X \\
X\end{array}$ & $\begin{array}{l}\mathrm{pH} \text { control with individual } \mathrm{O}_{2}, \mathrm{~N}_{2} \text {, } \\
\text { and } \mathrm{CO}_{2} \text { gas supply to oxygenator }\end{array}$ \\
\hline $\begin{array}{l}\text { Blood gas analysis } \\
\text { HA } \\
\text { PV } \\
\text { Vena cava }\end{array}$ & $x$ & $\begin{array}{l}X \\
X \\
X\end{array}$ & $\begin{array}{l}\text { Monitoring of blood gases and } \\
\text { other critical parameters during } \\
\text { long term perfusion }\end{array}$ \\
\hline $\begin{array}{l}\text { Physiologic PV } \\
\text { saturation with one } \\
\text { oxygenator }\end{array}$ & & $x$ & $\begin{array}{l}\text { Prevention of hyperoxygenation } \\
\text { Reduction of vasoconstriction }\end{array}$ \\
\hline Online glucose sensor & & $x$ & Real-time glucose monitoring \\
\hline $\begin{array}{l}\text { Feedback controlled } \\
\text { infusions } \\
\text { Insulin/glucagon }\end{array}$ & & $x$ & $\begin{array}{l}\text { Automated correction of blood } \\
\text { glucose level within predefined } \\
\text { limits }\end{array}$ \\
\hline $\begin{array}{l}\text { Feedback controlled } \\
\text { dialysis system }\end{array}$ & & $x$ & $\begin{array}{l}\text { Metabolic waste removal } \\
\text { Acid-base balance } \\
\text { Control of sodium \& electrolytes } \\
\text { Hematocrit control }\end{array}$ \\
\hline Liver movement & & $x$ & Prevention of pressure necrosis \\
\hline $\begin{array}{l}\text { Continuous response } \\
\text { evaluation to } \\
\text { vasoactiva, insulin and } \\
\text { glucagon }\end{array}$ & & $x$ & Continuous viability assessment \\
\hline
\end{tabular}

e

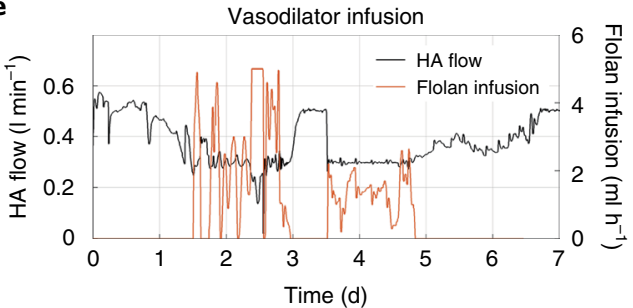

$\mathbf{f}$

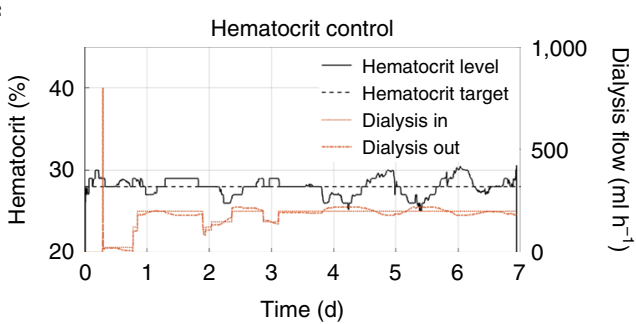

g

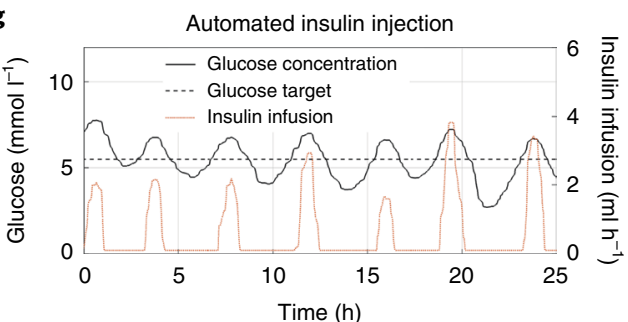


fixed on the edges inside the organ chamber. An inflatable balloon was positioned beneath the mat and connected to an air oscillator to induce oscillating movement (automated lifting and lowering) of the liver 15 times per minute (Supplementary Fig. 1). To test adequate perfusion after $7 \mathrm{~d}$ of ex vivo perfusion, we evaluated the distribution of the radioactive tracer 18 -fluorodeoxyglucose (FDG), as an indicator of preserved metabolism and viability inside the liver with positron emission tomography combined with computed tomography (PET-CT). This approach demonstrated homogeneous metabolism and iodinated contrast medium distribution with absence of non-perfused areas in contact places to the silicon mat (Fig. 2k).

Early phase of reperfusion of pig livers after 7-d perfusion. We tested the viability of eight pig livers perfused with autologous blood after 1 week of perfusion without exchanging the perfusate or adding any blood products. All livers showed preserved liver functions with production of bile flow over the entire perfusion period (Supplementary Fig. 3). While we observed an initial temporary increase of injury markers such as aspartate aminotransferase (AST) and 8-hydroxydesoxyguanosin (8-OHdG) within the first $12 \mathrm{~h}$ of perfusion, these values declined in the following days (Supplementary Fig. 3). Cytochrome C, an established marker for mitochondrial membrane injury, did not increase over the 7 - $d$ period and histology after 7 - $d$ of perfusion revealed no relevant necrosis or features of endothelial activation (Supplementary Figs. 3 and 4 ).

To test in vivo reperfusion injury and viability after a 7 -d perfusion, we transplanted three livers in recipient pigs of similar size. As controls, we also transplanted five livers that had been kept only for $2-3 \mathrm{~h}$ with static cold storage before transplantation ${ }^{22}$. The aim of these experiments was to confirm feasibility of vascular anastomoses and to investigate the early phase of reperfusion after long-term perfusion. All transplant experiments were terminated $3 \mathrm{~h}$ after transplantation under general anesthesia because survival experiments with cessation of general anesthesia were prohibited by our local animal protection authorities. Therefore, the main endpoints of these transplantation experiments remained early markers of reperfusion injury, including release of transaminases and histology. These results indicated no difference between release of liver enzymes and histology as compared to controls livers that had been transplanted after standard cold storage without long-term preservation in the machine perfusion system ${ }^{22}$ (Supplementary Fig. 5).
Seven-day perfusion and assessment of human livers. Next we applied the technology to human livers. Although pig and human livers have similar size and architecture, several substantial adjustments were necessary to successfully perfuse human livers. First, in contrast to pig liver perfusion with autologous fresh donor pig blood, the perfusate used for human livers consisted of preserved packed human blood products with altered electrolyte levels (for example, high potassium $(>10 \mathrm{mmol})$ and low $\mathrm{pH}(<7.0))$. Thus, electrolyte levels and $\mathrm{pH}$ were corrected through an integrated dialysis before starting perfusion. Second, pig and human livers disclosed differences in the sensitivity toward glucagon, with the need to adjust glucagon levels $\left(0.11 \mathrm{U} \mathrm{ml}^{-1}\right.$ in human versus $0.01 \mathrm{U} \mathrm{ml}^{-1}$ in pigs). Third, the vasodilator nitroprussiat used in pigs is not approved for human use in Switzerland and was therefore replaced by epoprostinolium (Flolan, GlaxoSmithKline) ${ }^{11}$. Fourth, the final human protocol includes ursodeoxycholic acid, which is commonly used in the clinic, in place of taurocholic acid derivatives for bile flow stimulation.

We perfused ten human livers that had been declined for transplantation by all hospitals in Switzerland, then in all Europe owing to poor quality, and as such were to be discarded (Supplementary Table 1). We connected the human livers to the ex vivo perfusion machine through the hepatic artery, portal vein, vena cava and bile duct. The perfusion was conducted using a blood-based perfusate, reconstituted from packed erythrocytes, fresh frozen plasma, albumin and platelets (Supplementary Table 2) at $34^{\circ} \mathrm{C}$ owing to protective effects at this temperature ${ }^{23}$.

In standard clinical transplantation, organs are preserved at about $4{ }^{\circ} \mathrm{C}$ under anaerobic conditions for up to $12-18 \mathrm{~h}$ before implantation. Many of these livers suffer additionally from prepreservation injury (that is, injury occurring before recovery of the organ owing to, for example, instability of the donor before death or medical treatment in intensive care units) and ischemia-reperfusion injury at the time of preservation and transplantation ${ }^{24}$. Consequently, cell death $(\sim 15 \%)$ is observed in most post-transplant biopsy specimens, even under optimal clinical conditions ${ }^{25,26}$. Similar to clinical transplantation, ischemia-reperfusion injury is unavoidable in machine-perfused livers when connecting the liver to the machine after cold storage and subsequently perfusing with oxygenated perfusates. To assess the livers during machine perfusion, we measured a number of parameters. In the perfusate, we monitored hepatocellular enzymes (alanine aminotransferase (ALT) and AST), signaling

Fig. 2 | Step-by-step integration of the components for long-term perfusion using pig livers. a, Amount of glycogen per milligram of liver tissue according to the glucose-level control method. In the hyperglycemic group, hyperglycemia $\left(>10 \mathrm{mmol} \mathrm{I}^{-1}\right)$ in the perfusate was maintained by constant glucose infusion ( $n=4$ pig livers). In the normoglycemic group, a targeted glucose level of $4 \mathrm{mmoll}^{-1}$ was maintained by manual adjustment of the glucose infusion ( $n=4$ pig livers). In the automated control group, glucose was never infused but was rather released from the liver by gluconeogenesis during a basal insulin injection. Glucagon was injected, if the desired glucose level was undershot after insulin injection and spontaneous glucose level recovery was not sufficient ( $n=5$ pig livers). b, Representative experiment from the normoglycemic group ( $n=4$ pig livers) demonstrating the complete glucose uptake (red circle) by hepatocytes under a high-dose ('fed state') insulin injection. c, Representative hematoxylin and eosin staining showing histological changes according to the glucose-level control method (top). Normal histology at perfusion start (middle). Enlarged, pale hepatocytes with excessive glycogen stores resembling glycogen-storage disease on day 3 with a 'hyperglycemic protocol' ( $n=4$ pig livers; bottom). Liver integrity on day 7 with the automated glucose metabolism control ( $n=5$ pig livers). Scale bars: overview, $500 \mu \mathrm{m}$; higher magnification, $100 \mu \mathrm{m}$ d, Hemolysis rate with ( $n=8$ pig livers) and without ( $n=8$ pig livers) pulsatile flow in the hepatic artery. e, Perfusate sodium level with ( $n=5$ pig livers) and without ( $n=4$ pig livers) dialysis. f. Hematocrit level maintenance with dialysis for $7 \mathrm{~d}(n=5$ pig livers). $\mathbf{g}$, BUN level course in perfusate with $(n=5)$ and without $(n=4)$ dialysis. h, Oxygen saturation in liver outflow (vena cava) according to perfusion with 'arterial blood' ( $n=5$ pig livers) and 'venous blood' ( $n=5$ pig livers) in the portal vein. i, The need for vasodilators during perfusion for $7 \mathrm{~d}$ with 'arterial' ( $n=5$ pig livers) and 'venous' blood ( $n=5$ pig livers) in portal vein. Increased use of vasodilators in the arterial portal vein implies increased resistance of the hepatic artery. $\mathbf{j}$, Representative experiments illustrating pressure necrosis in static storage and its prevention with liver movement (left). Early perfusion experiments with macroscopic pressure necrosis ( $n=2$ pig livers; middle left). Impaired circulation in pressure areas demonstrated by fluorescein distribution (red circle) ( $n=1$ pig liver; middle right and right). In a later developmental phase, perfusion was performed in situ ( $n=2$ pig livers). In this experimental setting, diaphragm movement was mimicked during isolated in situ liver perfusion with a medical ventilator after the animals were euthanized, whereupon the livers showed no pressure areas. Dark areas in the image to the right correspond to biopsy spots. k, Representative image showing radioactive glucose uptake in PET-CT after 1 week of perfusion showing homogenous metabolism without necrosis in contact areas to silicon mat ( $n=4$ pig livers). Data are reported as mean \pm s.d. For comparison of two groups a two-tailed Student's $t$ test was used. ${ }^{\star} P<0.05,{ }^{\star \star} P<0.01,{ }^{\star \star \star} P<0.001$; exact $P$ values are provided in Supplementary Table 3. NS, not significant. 
proteins (damage-associated molecular patterns (DAMPs)), uric acid, the proinflammatory cytokine interleukin-6 (IL-6), the antiinflammatory cytokine interleukin-10 (IL-10) and clearance of ammonia and lactate. We also measured liver tissue energy sources, such as adenosine triphosphate (ATP) and glycogen in tissue (measurement details are provided in the Methods). We also obtained daily liver tissue samples for histology. The liver sections were stained with hematoxylin and eosin and periodic acid Schiff (PAS) stain, and with antibodies against von Willebrand factor, phosphohistone 3 (pH3), CD68, cleaved caspase 3, Ki-67 and cytokeratin 7 (Methods).
Injury markers and DAMPs. We found that six of the livers, which we numbered 1 to 6 , demonstrated a decrease in injury and inflammation markers and DAMPS (ALT, AST, IL-6, IL-10 and uric acid) usually occurring after day 1 or 2 after perfusion, whereas the other four livers (numbered 7 to 10) failed to show any improvement in terms of injury and inflammation markers, as well as DAMPS (Fig. 3a-d and Supplementary Fig. 6). Livers 1 to 6 were maintained viable for the targeted time period of 1 week, while livers 7 to 10 failed to reach this objective, showing ongoing cell death and signs of liver failure. This required a premature termination of the experiment with a lack of data for perfusion days 5 to 7 . a
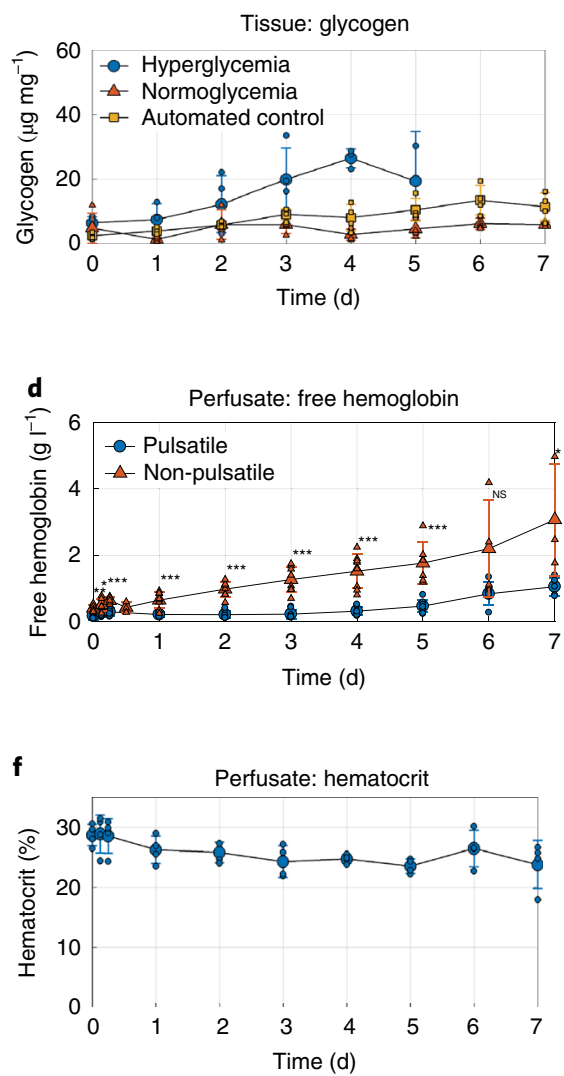

h

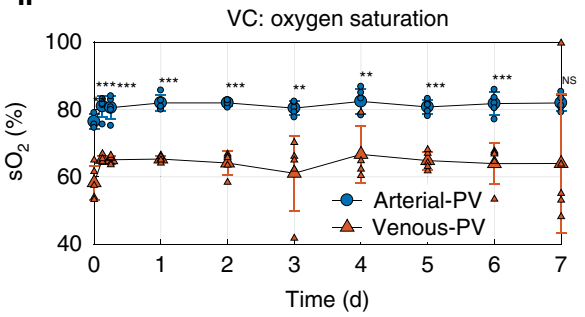

b

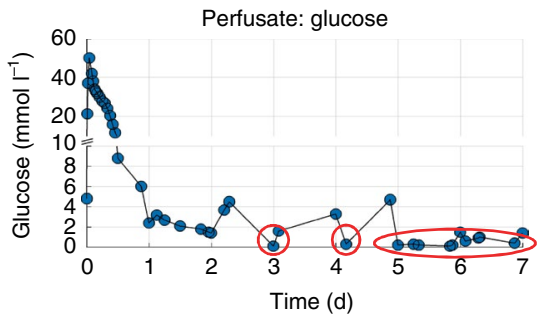

e

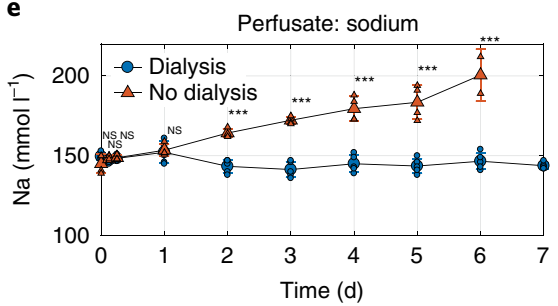

g
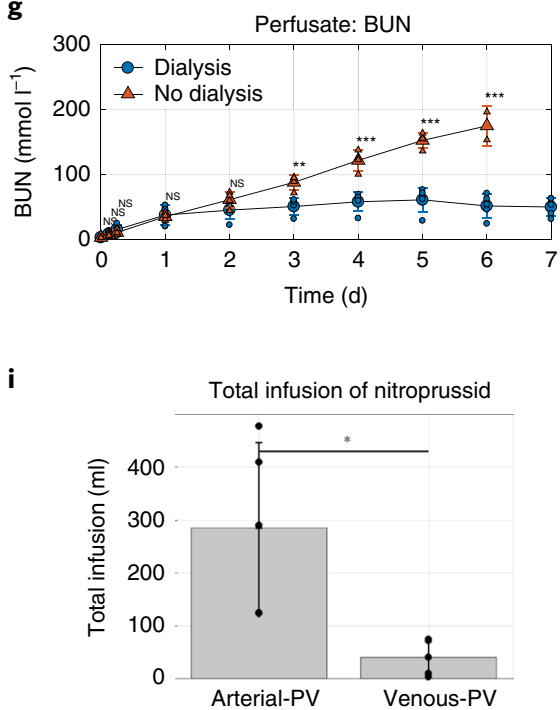

C Histology at start in hyperglycemic group

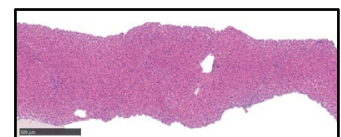

Excessive glycogen with hyperglycemia

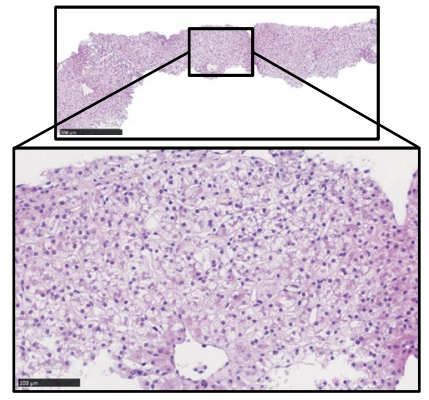

Integrity on day 7 with automated control

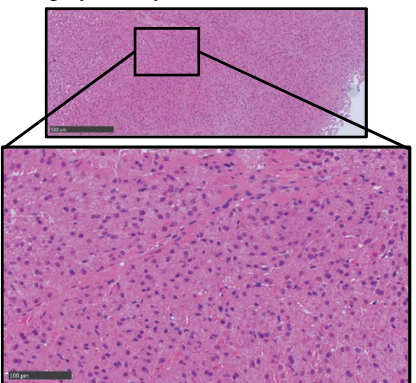

k

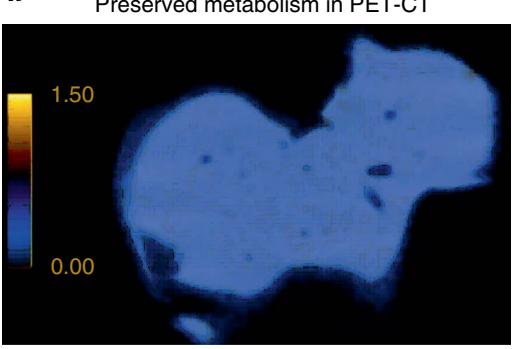

j Pressure areas without and with movement
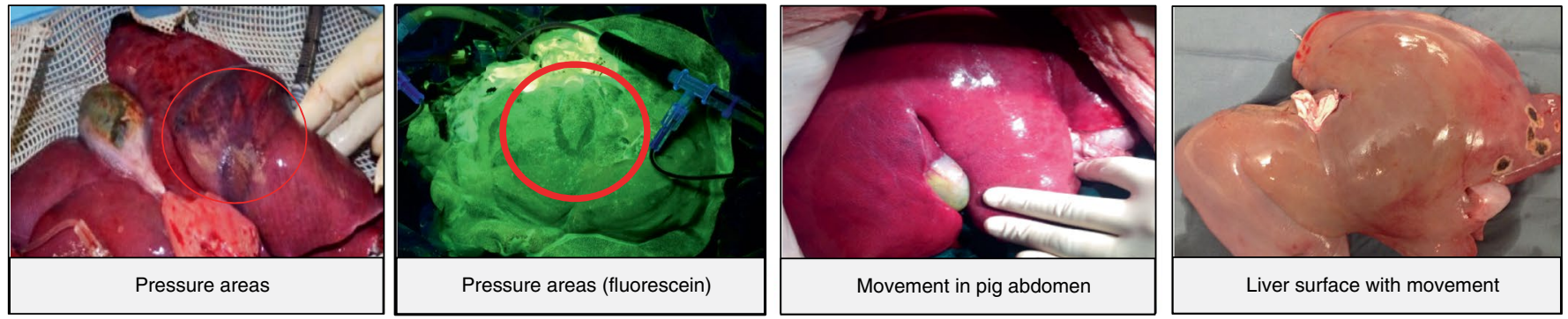


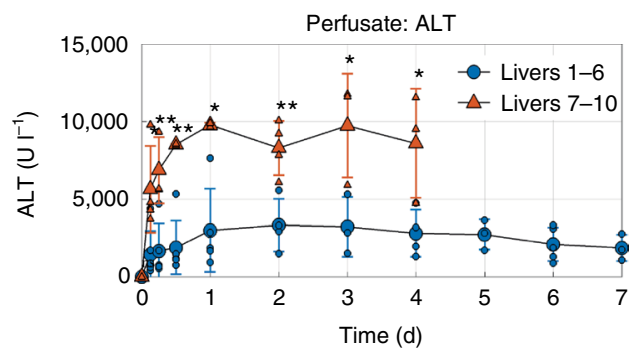

b
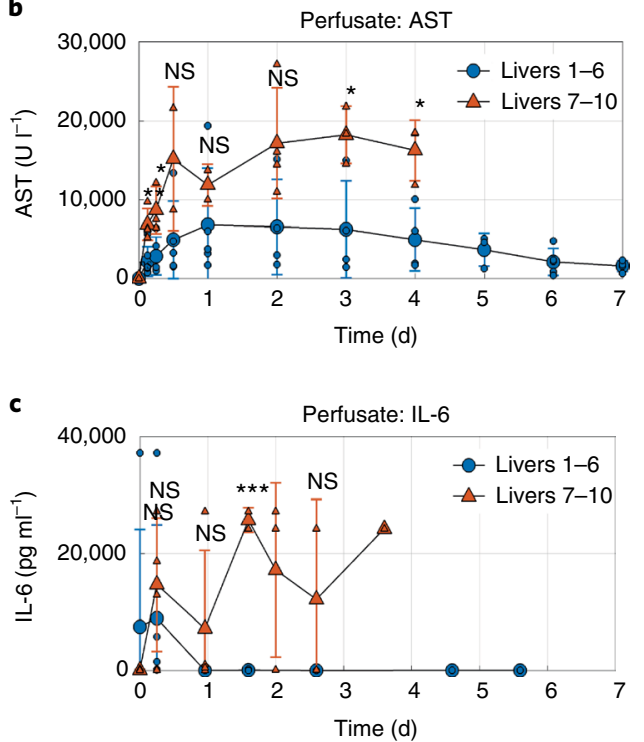

d

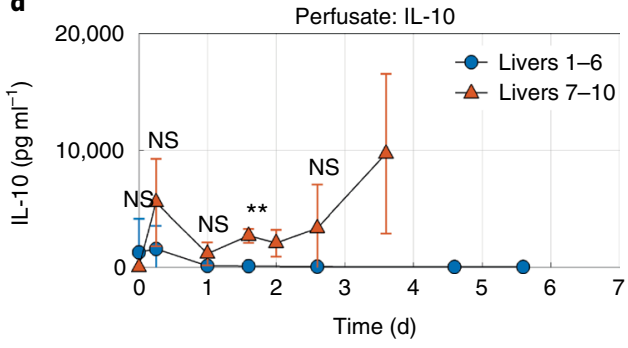

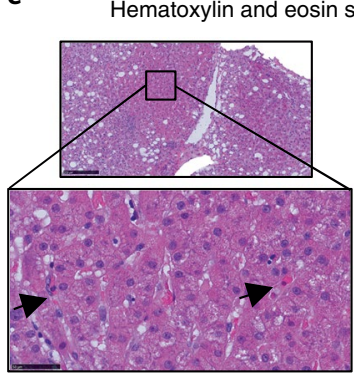

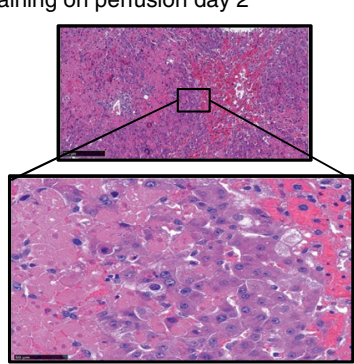

$\mathbf{g}$
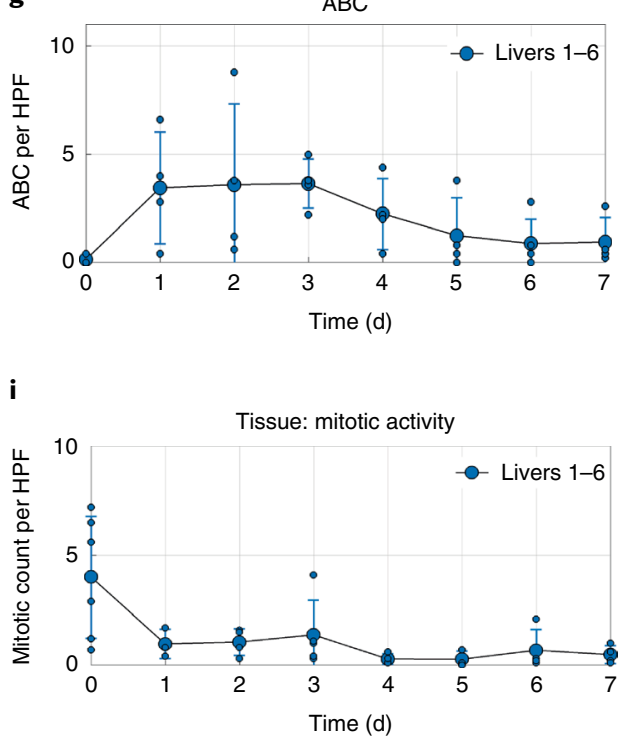

k

Preserved metabolism in PET-CT

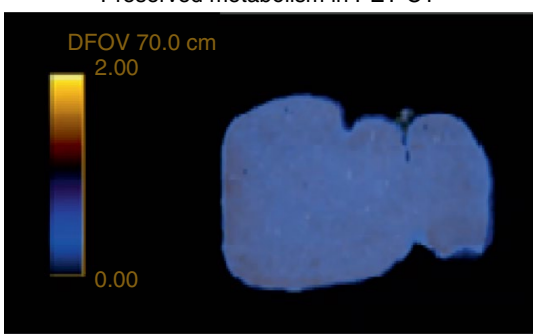

f Ex vivo engulfment

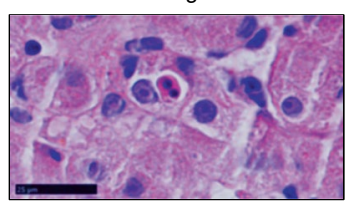

h Ex vivo phagocytosis

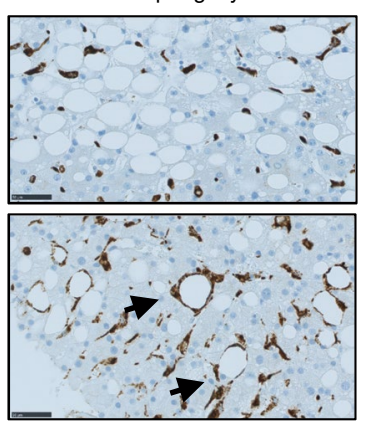

j

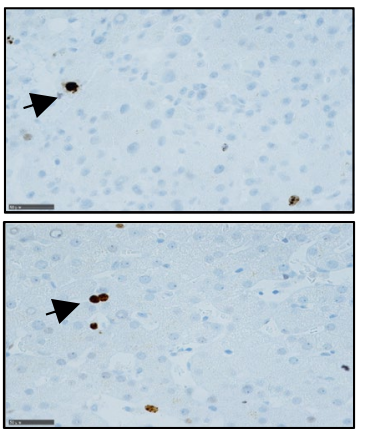

Fig. 3 | Injury markers in perfusate and tissue during ex vivo human liver perfusion ( $n=10$ livers). $\mathbf{a}, \mathbf{b}$, Release of injury markers into perfusate with ALT (a) and AST (b) levels. Human livers 1 to 6 (blue line, $n=6$ livers) and human livers 7 to 10 (red line, $n=4$ livers). c,d, Cytokine release shown for proinflammatory IL-6 (c;n=9) and anti-inflammatory IL-10 (d; $n=9)$. Error bars for livers 1 to 6 are not plotted on the presented scale. e, Hematoxylin and eosin staining on day 2. Left, representative hematoxylin and eosin staining showing apoptotic bodies (black arrows) in livers 1 to 6 . Right, representative hematoxylin and eosin staining showing massive cell death in livers 7 to 10; the higher magnification shows some hepatocytes that are still viable. Scale bars: slide overviews, $250 \mu \mathrm{m}$; higher magnifications, $50 \mu \mathrm{m}$. f, Representative image showing engulfment of an apoptotic body by hepatocytes (black arrow) in livers 1 to $6\left(\right.$ ref. ${ }^{40}$ ). Scale bar, $50 \mu \mathrm{m}$. g, Apoptotic body count (ABC) seen on hematoxylin and eosin staining (per HPF) in livers 1 to $6, \mathbf{h}$, Top, representative slides showing phagocytosis with $\mathrm{CD} 68^{+}$immunohistochemistry for liver macrophages (livers 4 and 5). Bottom, before reperfusion, fat vacuoles are not phagocytized. Phagocytosis of released fat vacuoles by macrophages (lipopeliosis) on day 4 in a steatotic liver (black arrows). Scale bar, $50 \mu \mathrm{m}$. i, Mitotic count ( $\mathrm{pH}^{+}$hepatocytes) per HPF in livers 1 to 6 . j. Representative slides from two grafts demonstrating nuclei of $\mathrm{pH} 3^{+}$hepatocytes (seen only during mitosis) on day 4 (black arrows) from livers 1 to 6 . Scale bars, $50 \mu \mathrm{m}$. k, Representative image demonstrating radioactive glucose uptake in PET-CT as a sign of preserved metabolism after 1 week of perfusion in a human liver (standardized uptake value $(S U V)_{\max } 1.15$, SUV $V_{\operatorname{mean}} 0.64$ ). Injected dose of $22 \mathrm{MBq}$ of radioactive glucose and uptake time of $78 \mathrm{~min}$. Notably, there was no sign of necrosis on areas of contact with the silicon mat in the PET-CT images $(n=1$, liver 6$)$. Data are reported as mean \pm s.d. For comparison of two groups two-tailed Student's $t$ test was used. ${ }^{\star} P<0.05,{ }^{\star \star} P<0.01$, ${ }^{\star \star \star} P<0.001$; exact $P$ values are provided in Supplementary Table 3.

Tissue injury assessed by histological analysis. The histological analysis of the livers before insertion into the perfusion machine revealed cirrhosis $(n=1)$, fibrosis grade $2-3(n=3)$, macrosteatosis $(>25 \%, n=1 ;>50 \%, n=1)$, inflammatory infiltrates $(n=4)$ and necrosis $(n=1)$ (Supplementary Table 1). In livers 1 to 6 , we observed injury until the second perfusion day, as assessed by hematoxylin and eosin staining (Fig. 3e-g). However, the number of non-viable cells declined continuously to reach the lowest level 


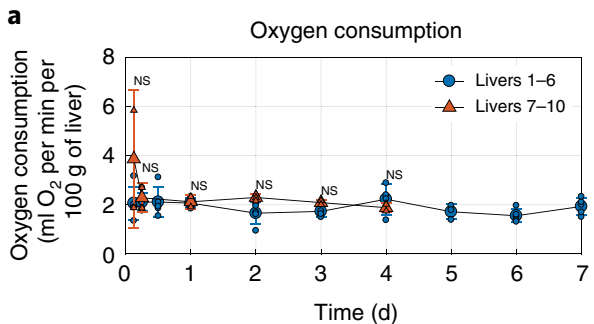

d

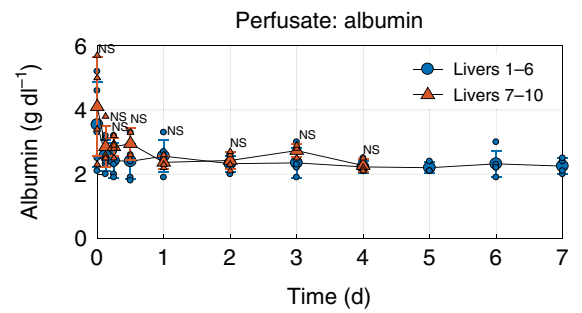

g

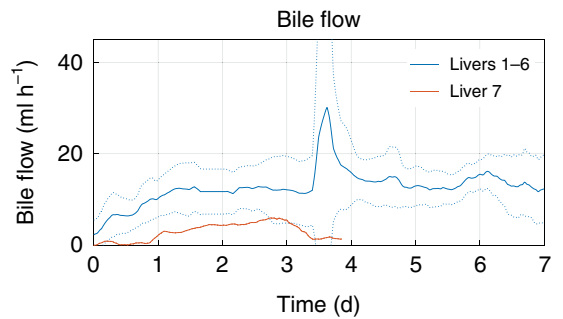

b

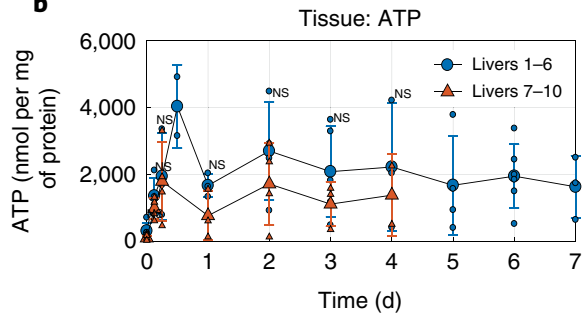

e

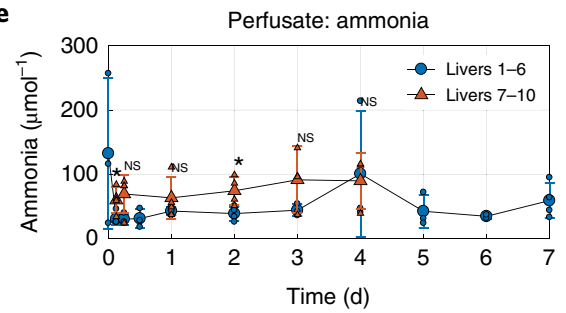

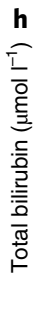

j

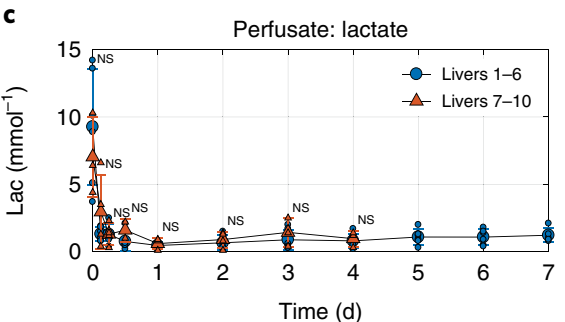

f
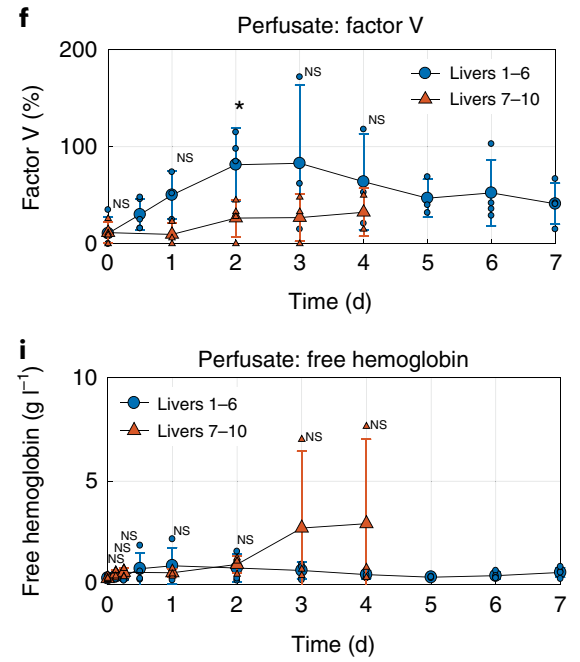

k

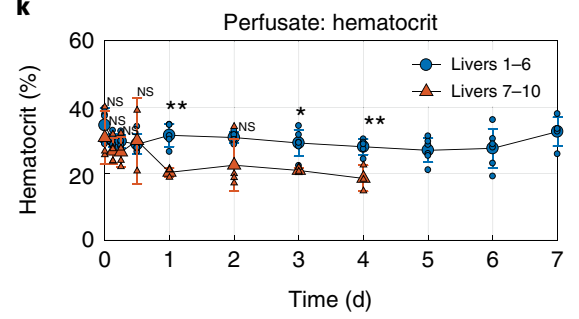

Fig. 4 | Liver function during human liver perfusion ( $\boldsymbol{n}=\mathbf{1 0}$ livers). $\mathbf{a}, \mathbf{b}$, Oxygen consumption (a), and ATP levels (b). Human livers 1 to 6 (blue line, $n=6$ livers), human livers 7 to 10 (red line, $n=4$ livers). No significant difference in liver functions was observed between the groups. c, Human blood products have high lactate at delivery (0 time). Lactate was cleared shortly after perfusion start by all the livers. d-f, Livers maintained a physiologic albumin level (d), cleared ammonia (e) and produced coagulation factor $\vee(\mathbf{f})$. $\mathbf{g}$, Bile flow was present constantly in livers 1 to 6 , while in livers 7 to 10 only one liver disclosed bile flow (data shown are mean (solid blue lines) with s.d. (dotted blue lines) for livers 1 to 6 ). $\mathbf{h}$, Clearance of bilirubin into bile. $\mathbf{i}$, Hemolysis rate. Free hemoglobin in livers 1 to 6 was maintained at a low level ( $n=4$ human livers) or was reduced during perfusion ( $n=2$ human livers). Livers 7 to 10 showed, although not significantly, increasing levels of free hemoglobin. Small error bars for livers 1 to 6 were not plotted on the presented scale after day 4. j, There was no macroscopic sign of hemolysis on a representative image of the daily centrifuged perfusate (plasma) from livers 1 to 6 . $\mathbf{k}$, Constant hematocrit level for $7 \mathrm{~d}$ without exchanging perfusate. Data are reported as mean \pm s.d. For comparison of two groups two-tailed Student's $t$ test was used. ${ }^{\star} P<0.05,{ }^{\star \star} P<0.01,{ }^{\star \star \star} P<0.001$; exact $P$ values are provided in Supplementary Table 3.

of $0.95 \pm 1.1$ per high-power field (HPF; $\times 40$ magnification) on day seven. In steatotic livers we observed clearance of fat, pointing to lipopeliosis as a sign for viable and functionally active macrophages (Fig. 3h). During perfusion, mitotic cells were also documented as a measure of preserved cell proliferation capacity ex vivo (Fig. 3i,j). After 1 week of perfusion, liver biopsies in livers 1 to 6 did not show evidence of substantial cell death (Supplementary Fig. 6). A PET-CT demonstrated preserved metabolism with absence of relevant nonperfused areas in places of contact with the silicon mat after 1 week of perfusion (Fig. 3k). With respect to total glycogen content of tissue we did not see a statistical difference between livers 1 to 6 and 7 to 10 . However, PAS staining detected an unequivocal glycogen loss in non-viable areas in livers 7 to 10 as compared to homogenous staining in livers 1 to 6 . These findings suggest an unequal qualitative glycogen distribution in livers 7 to 10 without a change in total glycogen amount as compared to livers 1 to 6 (Supplementary Fig. 6).

By contrast, hematoxylin and eosin staining of livers 7 to 10 showed higher injury, accompanied by low glucose levels in the perfusate at perfusion termination (Fig. 3e). Continuous substitution of glucose was necessary in these livers to maintain physiological perfusate glucose levels, similar to severe liver failure in clinical cases.

Livers 1 to 6 generally had endothelial cells with intact structure as assessed by immunohistochemistry of von Willebrand factor and mRNA level detection of intercellular adhesion molecule 1 (ICAM1) (Supplementary Fig. 6). By contrast, livers 7 to 10 generally showed activation of von Willebrand factor in areas of necrosis and elevated ICAM1 mRNA levels (Supplementary Fig. 6). 


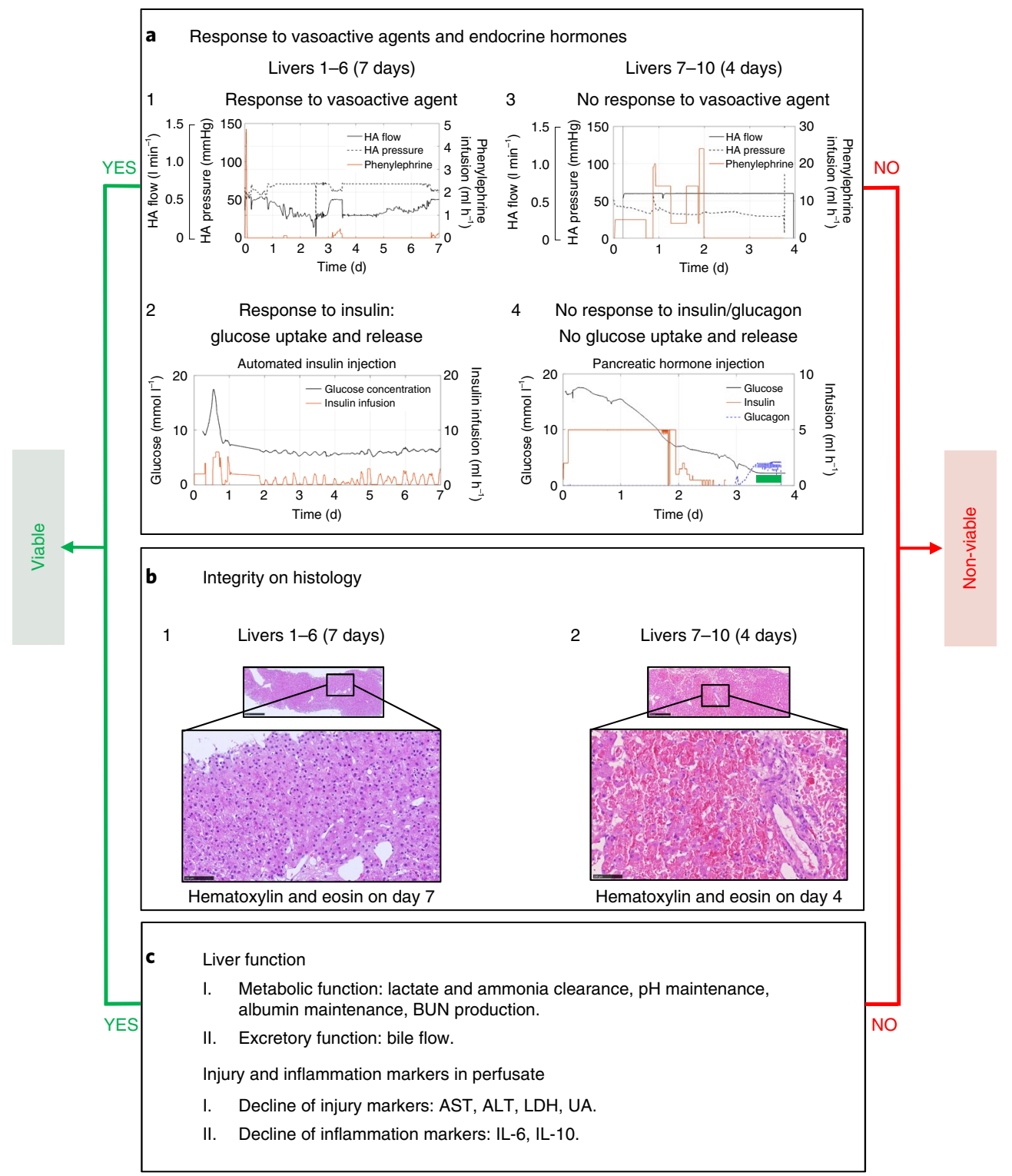

Fig. 5 | Multistep approach for viability assessment of preinjured human livers during long-term perfusion. a, Evaluation of liver response to vasoactive agents and pancreatic hormones. (1) Liver 1 to 6 , hepatic-artery vasculature maintains targeted hepatic-artery flow and pressure with response to vasoconstrictor (phenylephrine). (2) Livers 1 to 6 , decline of glucose level in perfusate as a response to insulin application and increase during minimal basal infusion. (3) Livers 7 to 10, vasoplegia with lack of hepatic-artery vasculature response to the vasoconstrictor. Despite injection of high doses of phenylephrine at the maximal continuous flow rate, the targeted hepatic-artery pressure cannot be maintained. (4) Livers 7 to 10, during perfusion, hyperglycemia changed to hypoglycemia, which was not responsive to glucagon injection. Glucose had to be substituted to maintain glucose levels in the perfusate within the normal range (green rectangle). b, Integrity in histology. (1) Livers 1-6 ( $n=6$ livers), representative hematoxylin and eosin staining demonstrating the preserved integrity without relevant cell death on day 7. (2) Livers 7-10 ( $n=4$ livers), representative hematoxylin and eosin staining with massive cell death on day 4 with loss of integrity. Scale bars: overview, $500 \mu \mathrm{m}$; higher magnification, $100 \mu \mathrm{m}$. c, Evaluation of liver function and injury markers: Livers with massive cell death might maintain some of the liver functions including, for example, lactate clearance. Therefore, we consider these listed liver functions only as viability markers in combination with adequate response to hormones and vasoactive substances, as well as histology. Additionally, it should be noted that the kinetics of cytokine and injury marker release are more decisive than peak values, as absolute levels are dependent on liver mass and perfusate volume.

All livers lost weight during ex vivo perfusion $(27.7 \pm 7.7 \%$; Supplementary Table 1).

Liver function. All perfused livers demonstrated considerable biological functions during ex vivo perfusion (Fig. 4). They continuously consumed oxygen (Fig. 4a), with increasing cellular ATP synthesis as compared to baseline values (Fig. 4b). Also, BUN production (Supplementary Fig. 6), lactate clearance (Fig. 4c) and maintenance of albumin level were present in all livers (Fig. 4d).
Nine livers showed ammonia clearance and synthesis of coagulation factor V (Fig. 4e,f), and seven of ten livers produced bile continuously (Fig. 4g), with effective bilirubin clearance (Fig. 4h). The mean blood $\mathrm{pH}$ was maintained between 7.2 and 7.5 (Supplementary Fig. 6). The perfusate was never exchanged during the entire course of perfusion in all cases (Fig. 4i-k). Additional blood products were not added except for liver 5, where there was substantial loss of the perfusate owing to a leak in the oxygenator. 
Hemodynamic response to vasoactive substances. Under physiologic conditions, hepatic artery flow is tightly controlled by the autonomic nervous system and vasoactive substances, which are dependent on liver signals, whereas portal vein flow acts independently ${ }^{9}$. The hepatic artery response to vasoactive substances is therefore a valuable sign of organ viability ${ }^{9}$. With a flow of $11 \mathrm{~min}^{-1}$, the portal pressure could be maintained in physiological range $(<10 \mathrm{mmHg})$ in each liver except in two livers (livers 1 and 3 ) that had pre-existing fibrosis and cirrhosis (portal vein pressure $12-15 \mathrm{mmHg}$ ), a typical feature observed in the clinic with patients suffering from injured parenchyma. For livers 1 to 6 , flow rates in the hepatic artery remained constant with infusion of vasoactive substances (Figs. 1e and 5a). By contrast, livers 7 to 10 became unresponsive to even high amounts of vasoconstrictors (vasoplegia) shortly after initiating perfusion $(n=1)$ or on day $4(n=2)$. This vasoplegia translated into a pressure of less than $45 \mathrm{mmHg}$ in the hepatic artery despite high vasoconstrictor infusion at a hepatic artery flow of $0.61 \mathrm{~min}^{-1}$ (Fig. 5a and Supplementary Fig. 7).

\section{Discussion}

In this study, we developed a perfusion machine preserving pig and human livers for 1 week with consistently good outcomes as assessed by intact liver structure on histology and preserved function as indicated by bile production, synthesis of blood proteins, maintenance of cellular energy (ATP), as well as an appropriate response to pancreatic hormones or vasoactive drugs. Because of restrictions by our local animal protection authorities, we were not able to assess transplantation efficacy beyond the time of extubation, but observation for a few hours after reperfusion showed no differences between livers perfused for 1 week as compared to the control group transplanted after a standard short period of cold storage.

The human livers available for this inaugural study were severely injured and heterogeneous with respect to cold and warm ischemia times, underlying liver diseases, donor age, duration of intensive care unit treatment among other parameters (Supplementary Table 1). Because of an organ shortage in our country, a control group of less injured livers is not available, as such livers are consistently used for transplantation ${ }^{27,28}$. The heterogeneity and poor quality of the livers poses difficulties in assessing the outcome overall. However, the many measurements obtained throughout the 1-week perfusion at $34^{\circ} \mathrm{C}$ suggest that many of the livers maintained viability and to some extent recovered from prepreservation and initial ischemiareperfusion injuries following connection to the machine.

In addition, several parameters of viability remain difficult to assess, such as the long-term viability of intrahepatic bile ducts. The development of bile duct strictures occurs in some grafts up to 1 year after transplantation, typically as a result of prolonged warm ischemic injury ${ }^{29}$. Previous studies suggested glucose clearance in bile as a sign of cholangiocyte viability ${ }^{4,30}$, proposing a bile/perfusate glucose ratio less than 0.7 as a cut off for predicting viability ${ }^{30}$. Notably, these criteria were all met in our perfused livers (Supplementary Fig. 8). The final proof of bile duct integrity, however, can only be assessed after transplantation of perfused livers in recipients with at least a 1-year follow-up ${ }^{29}$. Similarly, coagulation, an additional typical parameter of liver synthesis ${ }^{31}$, was difficult to monitor during ex vivo perfusion. Interpretation of factor $\mathrm{V}$ and international normalized ratio (INR) during ex vivo perfusion is limited owing to the heparinised circuit $^{32}$.

Therefore, we interpreted liver function by a combination of ATP synthesis, bile flow, ammonia clearance and BUN production, as well as by the need for glucose substitution, which is another well-known marker of irreversible hepatocellular injury. These parameters were combined with the response of the livers to added vasoconstrictors, as failing liver grafts are frequently characterized by severe vasoplegia, requiring high dosages of vasoconstrictors to maintain adequate blood flow in the clinical setting ${ }^{33}$. Some livers became unresponsive to vasoconstrictors during the perfusion course. The reason for failure in those livers probably relates to excessive warm or cold ischemia times, in combination with prepreservation injury recognized in the clinic as extended graft criteria, enhancing severe ischemia-reperfusion injury ${ }^{17}$. In Fig. 5, we summarize our current understanding of how to best assess liver function and viability during ex vivo perfusion. Besides the conventional parameters (that is, lactate clearance, perfusate $\mathrm{pH}$ and bile production) as previously proposed ${ }^{4,34-36}$, we rely on the response to glycolytic hormones, vasoactive drugs and on clearance of ammonia, as well as histology and ATP synthesis.

Bile production, which is one of the most convincing indicators of liver viability after transplantation ${ }^{31}$, was present in the majority of livers perfused for 1 week.

Some of the human livers perfused for 1 week displayed a course of laboratory parameters and histology that was similar to those observed in the clinical setting of liver transplantation. For example, as in some of the livers perfused for 1 week, AST and ALT levels peak at around day 1, likely owing to some hepatocyte injury upon reperfusion, but decline rapidly thereafter similar to successful clinical liver transplantation ${ }^{31,37}$. Likewise, blood bilirubin increased during ex vivo perfusion with a delay in some livers as compared to liver enzymes ${ }^{37}$.

We did observe that the perfused livers consistently lost weight during the $7-d$ perfusion period, reaching about $25 \%$ of the initial graft mass. While the underlying mechanisms and clinical relevance of this finding remains unclear, we speculate that this phenomenon is largely related to recovery of mainly cellular swelling in injured livers, and therefore without negative impact on hepatic function. Similarly, the well-established circadian liver weight fluctuation has no negative impact on hepatic function ${ }^{38}$. Of note, we did not observe any weight change when using healthy pig livers (preperfusion weight of $1.7 \pm 0.03 \mathrm{~kg}$, post-perfusion weight $1.6 \pm 0.1 \mathrm{~kg}$ ). A similar loss of liver weight without substantial impact on function has been observed during short-term normothermic perfusion of livers from donations after circulatory death or steatotic liver grafts ${ }^{12}$.

We grouped the livers on the basis of organ viability and preserved integrity on histology on 1 -week perfusion. Livers 7 to 10 failed to reach the targeted perfusion duration developing signs of progressive liver failure, which required premature termination of perfusion. The observation of failure to respond to hormones and vasoactive drugs, loss of integrity on histology, as well as the absence of decline in injury and inflammation markers in perfusate in failed livers, offered a solid base to assess viability in long-term liver perfusion. These parameters may be advantageous over conventionally used liver function parameters in the clinic and in selection of grafts for transplantation (Fig. 5), which were not substantially different in our system. However, further studies will be needed to determine which are the best measures of liver viability after longterm perfusion.

Overall, the results in group 1 livers (livers 1 to 6 ) are promising and suggest that machine perfusion for 1 week may support viability of human livers, although testing with higher-quality livers and transplantation of perfused livers into recipients will be required to fully assess the potential of our approach.

In ischemia-reperfusion injury, reoxygenation of ischemic cells triggers a burst of reactive oxygen species, which are released mainly by mitochondria ${ }^{25}$. Consequently, machine perfusion strategies should target primarily mitochondrial metabolism ${ }^{39}$, for example, by applying membrane-permeable scavengers and reversible inhibitors of electron transfer, or by prevention of the opening of permeability transition pores. An ex vivo system offers the potential advantage of avoiding such undesirable systemic effects in the recipient. We envision our technology being used primarily with organs currently declined for transplantation, with long-term 
ex vivo perfusion under physiologic conditions potentially enabling the rescue of organs or their modification in a variety of ways. For example, 1-week perfusion may enable repair of severely steatotic livers, modification of the immunogenicity of the graft or recipient to induce tolerance, or treatment of tumors in livers using effective chemotherapeutic agents that are too toxic for systemic use. In addition, long-term perfusion would allow organs to be transported safely over long distances, improving worldwide organ sharing and logistics for transplantation in many centers ${ }^{1,3}$. Another opportunity in the future might be to enable liver regeneration, with a donor liver split into several anatomic segments to regrow in the machine. Recent surgical procedures have shown that massive liver regeneration in humans can occur within $6 \mathrm{~d}$ of major tissue loss ${ }^{7}$. If feasible, such an approach could double or even triple the availability of organs for transplantation. We may also imagine the use of partial grafts for auto-transplantation in patients with liver cancer, obviating the need for immune suppression.

In summary, this study introduces a perfusion technology developed by a group of surgeons, biologists and engineers that enables maintenance of injured human livers in a functional state for 1 week ex vivo. With further testing to demonstrate its use with injured livers and long-term transplantation success, this machine may provide an avenue for providing more patients with liver grafts.

\section{Online content}

Any methods, additional references, Nature Research reporting summaries, source data, extended data, supplementary information, acknowledgements, peer review information; details of author contributions and competing interests; and statements of data and code availability are available at https://doi.org/10.1038/s41587019-0374-x

Received: 23 December 2018; Accepted: 18 November 2019; Published online: 13 January 2020

\section{References}

1. Giwa, S. et al. The promise of organ and tissue preservation to transform medicine. Nat. Biotechnol. 35, 530-542 (2017).

2. de Vries, R. J. et al. Supercooling extends preservation time of human livers Nat. Biotechnol. 37, 1131-1136 (2019).

3. Buying time for transplants. Nat. Biotechnol. 35, 801 (2017).

4. Watson, C. J. E. et al. Observations on the ex situ perfusion of livers for transplantation. Am. J. Transplant. 18, 2005-2020 (2018).

5. Nasralla, D. et al. A randomized trial of normothermic preservation in liver transplantation. Nature 557, 50-56 (2018).

6. Eshmuminov, D. et al. Meta-analysis of associating liver partition with portal vein ligation and portal vein occlusion for two-stage hepatectomy. Br. J. Surg. 103, 1768-1782 (2016).

7. Schnitzbauer, A. A. et al. Right portal vein ligation combined with in situ splitting induces rapid left lateral liver lobe hypertrophy enabling 2-staged extended right hepatic resection in small-for-size settings. Ann. Surg. 255, 405-414 (2012).

8. de Santibanes, E. \& Clavien, P. A. Playing Play-Doh to prevent postoperative liver failure: the "ALPPS" approach. Ann. Surg. 255, 415-417 (2012).

9. Lautt, W. W. In Colloquium Series on Integrated Systems Physiology: from Molecule to Function to Disease (Eds. Granger, D. N. and Granger, J. P.) (San Rafael, 2009).

10. Berg, J. M, Tymoczko, J. L. \& Stryer, L. Biochemistry 5th edn (W. H. Freeman, 2002).

11. Eshmuminov, D et al. Perfusion settings and additives in liver normothermic machine perfusion with red blood cells as oxygen carrier. A systematic review of human and porcine perfusion protocols. Transplant Int. 31, 956-969 (2018).

12. Liu, Q. et al. Lipid metabolism and functional assessment of discarded human livers with steatosis undergoing $24 \mathrm{~h}$ of normothermic machine perfusion. Liver Transpl. 24, 233-245 (2018).

13. Baba, A. et al. Microcirculation of the bulbar conjunctiva in the goat implanted with a total artificial heart: effects of pulsatile and nonpulsatile flow. ASAIO J. 50, 321-327 (2004).
14. Orime, Y. et al. The role of pulsatility in end-organ microcirculation after cardiogenic shock. ASAIO J. 42, M724-M729 (1996).

15. O’Neil, M. P., Fleming, J. C., Badhwar, A. \& Guo, L. R. Pulsatile versus nonpulsatile flow during cardiopulmonary bypass: microcirculatory and systemic effects. Ann. Thorac. Surg. 94, 2046-2053 (2012).

16. Koning, N. J. et al. Pulsatile flow during cardiopulmonary bypass preserves postoperative microcirculatory perfusion irrespective of systemic hemodynamics. J. Appl. Physiol. 112, 1727-1734 (2012).

17. European Association for the Study of the Liver. EASL clinical practice guidelines: liver transplantation. J. Hepatol. 64, 433-485 (2016).

18. Attaye, I. et al. The effects of hyperoxia on microvascular endothelial cell proliferation and production of vaso-active substances. Intensive Care Med. Exp. 5, 22 (2017).

19. Dallinger, S. et al. Endothelin-1 contributes to hyperoxia-induced vasoconstriction in the human retina. Invest. Ophthalmol. Vis. Sci. 41, 864-869 (2000)

20. Liu, Q. et al. Ex situ 86-hour liver perfusion: pushing the boundary of organ preservation. Liver Transpl. 24, 557-561 (2018).

21. Neuhaus, P. \& Blumhardt, G. Extracorporeal liver perfusion: applications of an improved model for experimental studies of the liver. Int. J. Artif. Organs 16, 729-739 (1993).

22. de Rougemont, O. et al. One hour hypothermic oxygenated perfusion (HOPE) protects nonviable liver allografts donated after cardiac death. Ann. Surg. 250, 674-683 (2009).

23. Saad, H. \& Aladawy, M. Temperature management in cardiac surgery. Glob. Cardiol. Sci. Pract. 2013, 44-62 (2013).

24. Clavien, P. A., Harvey, P. R. \& Strasberg, S. M. Preservation and reperfusion injuries in liver allografts. An overview and synthesis of current studies. Transplantation 53, 957-978 (1992).

25. Chouchani, E. T. et al. A unifying mechanism for mitochondrial superoxide production during ischemia-reperfusion injury. Cell Metab. 23, 254-263 (2016).

26. Selzner, M., Rudiger, H. A., Sindram, D., Madden, J. \& Clavien, P. A. Mechanisms of ischemic injury are different in the steatotic and normal rat liver. Hepatology 32, 1280-1288 (2000).

27. McCormack, L. et al. Use of severely steatotic grafts in liver transplantation: a matched case-control study. Ann Surg. 246, 940-946 (2007).

28. Muller, $\mathrm{X}$ et al. Can hypothermic oxygenated perfusion (HOPE) rescue futile DCD liver grafts? HPB 21, 1156-1165 (2019).

29. Muller, X. et al. Defining benchmarks in liver transplantation: a multicenter outcome analysis determining best achievable results. Ann. Surg. 267, 419-425 (2018).

30. Matton, A. P. M. et al. Biliary bicarbonate, $\mathrm{pH}$, and glucose are suitable biomarkers of biliary viability during ex situ normothermic machine perfusion of human donor livers. Transplantation 103, 1405-1413 (2019).

31. Shaked, A., Nunes, F. A., Olthoff, K. M. \& Lucey, M. R. Assessment of liver function: pre- and peritransplant evaluation. Clin. Chem. 43, 1539-1545 (1997).

32. Karangwa, S. A. et al. Production of physiologically relevant quantities of hemostatic proteins during ex situ normothermic machine perfusion of human livers. Liver Transpl. 24, 1298-1302 (2018).

33. Neves, D. B., Rusi, M. B., Diaz, L. G. \& Salvalaggio, P. Primary graft dysfunction of the liver: definitions, diagnostic criteria and risk factors. Einstein 14, 567-572 (2016).

34. Laing, R. W. et al. Viability testing and transplantation of marginal livers (VITTAL) using normothermic machine perfusion: study protocol for an open-label, non-randomised, prospective, single-arm trial. BMJ Open 7, e017733 (2017)

35. Verhoeven, C. J. et al. Biomarkers to assess graft quality during conventional and machine preservation in liver transplantation. J. Hepatol. 61, 672-684 (2014)

36. Linares-Cervantes, I et al. Predictor parameters of liver viability during porcine normothermic ex situ liver perfusion in a model of liver transplantation with marginal grafts. Am. J. Transplant. 19, 2991-3005 (2019).

37. Fedoravicius, A. \& Charlton, M. Abnormal liver tests after liver transplantation. Clin. Liver. Dis. 7, 73-79 (2016).

38. Sinturel, F. et al. Diurnal oscillations in liver mass and cell size accompany ribosome assembly cycles. Cell 169, 651-663 (2017).

39. Dutkowski, P. \& Clavien, P. A. Uploading cellular batteries: caring for mitochondria is key. Liver Transpl. 24, 462-464 (2018).

40. Guicciardi, M. E. \& Gores, G. J. Apoptosis: a mechanism of acute and chronic liver injury. Gut 54, 1024-1033 (2005).

Publisher's note Springer Nature remains neutral with regard to jurisdictional claims in published maps and institutional affiliations.

(c) The Author(s), under exclusive licence to Springer Nature America, Inc. 2020 


\section{Methods}

Human livers. Ten human livers were obtained for research after being declined for transplantation nationwide and after consent was obtained from the next of kin. The local ethics committee of the Canton of Zurich approved the study protocol (2017-000412). The declined livers were procured in a standardized technique using IGL-1 for cooling and preservation. Some livers from donations after circulatory death were declined after initial hypothermic oxygenated liver machine perfusion for clinical purpose ${ }^{28}$ (Supplementary Table 1). During setup of the perfusion machine, the livers were prepared on the back table including removal of the gall bladder and cannulation of the hepatic artery, portal vein, vena cava and bile duct. Shortly before the start of liver perfusion, the preservation solution was flushed out with 21 of cold $\left(4^{\circ} \mathrm{C}\right)$ Ringerfundin solution (B. Braun Melsungen) through the portal vein (1.5l) and hepatic artery (0.5l).

Perfusion machine and protocol for human livers. Hemodynamic control. One centrifugal pump (Thoratec CentriMag) in combination with three pinch valves (Resolution Air MPPV-8) were used to maintain the desired flow or pressure conditions in the perfusion system. Flow sensors (Sonoflow CO.56) and pressure sensors (PendoTECH Single Use Pressure Sensors) measured flow and pressure in the respective silicon tube (RAUMEDIC) lines. Thereby, the control system aimed to maintain a desired flow (for example, $11 \mathrm{~min}^{-1}$ ) in the portal vein and the desired pressure conditions in the hepatic artery. To increase its resistance and provide the required flow rate ratio between the portal vein and hepatic artery, a pinch valve clamps the tube leading to the portal vein. In the hepatic artery, a pulsatile flow was applied targeting an MAP of at least $65 \mathrm{mmHg}$ (for example, systolic and diastolic pressure of $80 \mathrm{mmHg}$ to $50 \mathrm{mmHg}$ respectively with 60 beats per minute), while limiting the mean hepatic-artery flow to a maximum of $0.61 \mathrm{~min}^{-1}$. Pulsatile flow in the hepatic artery was realized by pulsatile operation (varying rotational speed) of the blood pump. Thereby, the rotational speed of the pump was defined in the same waveform as the desired arterial pressure pulse.

To prevent the flow in the hepatic artery from exceeding $0.61 \mathrm{~min}^{-1}$, the system injected the vasoconstrictor Neo Synephrine HCL (phenylephrine, Ospedalia) at an upper bound of $0.55 \mathrm{lmin}^{-1}$. On the other hand, to maintain a lower limit at least $0.251 \mathrm{~min}^{-1}$, the system injected the vasodilator Flolan (epoprostenol, GlaxoSmithKline). In both cases, this was realized by means of a proportional integral controller, defining the infusion rate on the basis of the measured hepaticartery flow rate.

Vena cava pressure control. Inadequate blood outflow from the liver leads to a vascular congestion and an ischemic hepatocellular damage. Thus, the system controlled the vena cava pressure around the atmospheric pressure (approximately $0 \mathrm{mmHg}$ ).

The vena cava was connected via tube set directly to the blood reservoir. By means of the height difference between the liver and the reservoir, a suction pressure was induced and additionally controlled through a pinch valve between the vena cava and the reservoir to control a desired vena cava pressure. The pinch valve induced an additional pressure drop, allowing elevation of the pressure at the vena cava to the desired levels. If the blood pressure inside the vena cava was lower than the surrounding ambient pressure, the vessel wall collapsed (usually around $0 \mathrm{mmHg}$ ), closed shortly and therefore induced a certain (negative) pressure fluctuation in the VC line. This induced pressure fluctuation was utilized in the control system to target the desired vena cava pressure. Finally, this pressure fluctuation (collapse of the vena cava) was detected by the pressure sensor in the vena cava line, with the control system reacting accordingly by closing the pinch valve in the vena cava line and thereby increasing the target vena cava pressure automatically by $1 \mathrm{mmHg}$, slightly above the fluctuation point. The system continuously monitored the minimum and maximum value of the vena cava pressure of the last $30 \mathrm{~s}$. If the difference between the minimum and maximum vena cava pressure in this time interval was greater than a certain threshold defined by the system (usually $10 \mathrm{mmHg}$ ), a fluctuation was detected. Afterward, the control system immediately increased the pressure in the vena cava line by a value of $1 \mathrm{mmHg}$, as described above. During normal operation, the system continuously searched for the fluctuation point. This was done by permanently decreasing the pressure set point for the vena cava by a rate of $1 \mathrm{mmHg}$ per hour. Once fluctuation had been detected, the set point was again increased using the algorithm described above, and the system again began to search for the fluctuation pressure. This control strategy was implemented owing to the fact that the pressure distribution inside the vena cava line depended on the flow rate and therefore the vena cava pressure was also flow-rate dependent. During the perfusion duration, the total flow rate through the vena cava line always varied over time. Therefore, a steady search for the fluctuation point was implemented (Supplementary Fig. 1).

Feedback-controlled injection of insulin and glucagon. An online glucose sensor (CITSens Bio Glucose Sensor, C-CIT Sensor) was integrated into the arterial line for real-time perfusate glucose measurement. The integrated sensor continuously measured the glucose in the perfusate. The blood glucose level was kept in a range defined by the user. If the blood glucose value surpassed the desired level, the system automatically injected insulin (Actrapid, Novo Nordisk Pharma), or, if the blood glucose undershot a lower threshold, the system injected glucagon
(GlucaGen, Novo Nordisk Pharma) in a closed-loop manner to maintain the perfusate glucose in a defined range. Both hormones can be kept at a basal rate as defined by the user. The injection rate of insulin was in the range of 0.02 to $4.5 \mathrm{IU} \mathrm{h}^{-1}$ depending on perfusate glucose level. The glucagon was injected at rates of 0.02 to $0.5 \mathrm{IUh}^{-1}$.

Blood-gas analysis and control. By the use of an oxygenator (Medos Hilite 2400 LT) and three individual gas-flow controllers (Bronkhorst Schweiz) for oxygen, nitrogen and $\mathrm{CO}_{2}$, the partial oxygen pressure $\left(\mathrm{pO}_{2}\right)$, partial $\mathrm{CO}_{2}$ pressure $\left(\mathrm{pCO}_{2}\right)$ and $\mathrm{pH}$ of the blood were controlled. An online blood-gas sensor (Terumo CDI 500 Shunt Sensor) continuously measured $\mathrm{pO}_{2}, \mathrm{pCO}_{2}$ and $\mathrm{pH}$. A proportional integral controller defined the oxygen concentration in the gas supply of the oxygenator to maintain $\mathrm{pO}_{2}$ at the desired level (10-12 kPa). Another proportional integral controller defined the total gas flow rate (oxygen and nitrogen) to control the wash-out rate of $\mathrm{pCO}_{2}$ in the perfusate. With $\mathrm{CO}_{2}$ being present in its dissolved form as carbonic acid in the blood, the $\mathrm{pCO}_{2}$ level could be manipulated to vary $\mathrm{pH}$ in a certain range. The $\mathrm{pCO}_{2}$ was allowed to vary from 4.5 to $6.5 \mathrm{kPa}$ to maintain a target $\mathrm{pH}$ of around 7.4 . With the inverse proportionality between $\mathrm{pCO}_{2}$ and $\mathrm{pH}$, an increasing $\mathrm{pCO}_{2}$ lead to a decrease in $\mathrm{pH}$, that is, the blood became more acidic. Therefore, to reduce the $\mathrm{pH}$, less $\mathrm{pCO}_{2}$ was washed out by reducing the total gas flow rate through the oxygenator, resulting in an increase of $\mathrm{pCO}_{2}$. The control was implemented by means of a control cascade with the inner control loop maintaining the secondary target $\mathrm{pCO}_{2}$ and the outer control loop maintaining the primary target $\mathrm{pH}$ of 7.4 by varying the $\mathrm{pCO}_{2}$ set point in the defined range of 4.5 to $6.5 \mathrm{kPa} . \mathrm{CO}_{2}$ was only supplied in the gas stream to the oxygenator before starting the liver perfusion and in the initial phase until the liver produced enough $\mathrm{CO}_{2}$ to maintain the target $\mathrm{pCO}_{2}$ in the blood. In the normal perfusion phase, a surplus of $\mathrm{CO}_{2}$ is produced by the liver, requiring it to be washed out.

Physiologic oxygen saturation in portal vein. In the in vivo situation, the portal vein originates from the intestinal organs and, therefore, contains less oxygen as compared to the hepatic artery. To mimic the physiologically reduced oxygen saturation in the portal vein, venous blood from the reservoir was mixed with a portion of arterial blood leaving the oxygenator. For this purpose, the $\mathrm{pO}_{2}$ was measured in the portal vein by means of an online blood-gas sensor (Shunt Sensor, Terumo, CDI 500) as stated above. In addition, the system continuously monitored the oxygen saturation in the vena cava $\left(\mathrm{vSO}_{2}\right)$ using a saturation sensor (CDI H/S Cuvette, Terumo, CDI 500). To mimic the physiological venous oxygen saturation in the portal vein, venous blood from the reservoir (blood from the vena cava with low, venous saturation) bypassed the oxygenator and was mixed with a portion of the arterial blood from the oxygenator to achieve desired saturation levels. The control was realized by means of a cascaded control. The inner control loop controlled the blood flow rate of the oxygenated blood (arterial blood) and the 'bypass flow rate' by means of a continuous pinch valve. The outer control loop controlled the oxygen saturation of the vena cava by varying the set point of the bypass flow rate. The goal was to keep a stable oxygenation level in the vena cava of $65 \%$ by varying the portal vein oxygen content. The total flow rate in the portal vein was maintained at $11 \mathrm{~min}^{-1}$. An increase of flow in the bypass was automatically compensated to maintain a constant total flow rate in the portal vein.

Feedback-controlled dialysis. A dialysis loop has been integrated into the perfusion system to supply and/or remove electrolytes, bicarbonates and metabolic waste products. By defining the TMF of fluid across the dialysis filter, dialysis was further utilized to control the blood hematocrit level by adding or removing fluid from the perfusion loop. A roller pump with multiple individual channels (Ismatec Reglo ICC) controlled the inflow and outflow of dialysate to the dialysis filter (Ultraflux AV-Paed, Fresenius Medical Care), thereby allowing the system to define the TMF. The targeted TMF was defined by the user or the controller of the system. Usually, the TMF was in the range of $-100 \mathrm{mlh}^{-1}$ to $100 \mathrm{mlh}^{-1}$. By controlling the TMF, the system can vary the amount of blood plasma in the perfusate and, thus, control the hematocrit level in perfusate. For this purpose, the control system used the continuous hematocrit measurement from the Terumo CDI 500 Cuvette sensor in the vena cava line.

Perfusate components and additives. The perfusate consisted of blood-groupmatched human red blood cells (approximately 1.41), fresh frozen plasma (approximately 0.81) and 1 unit of platelets from the human blood bank. A heparin bolus $(5000 \mathrm{U})$ was given at the start of perfusion. Albumin was added until it reached reference range $\left(>2.0 \mathrm{gl}^{-1}\right)$. As soon as the tube set was primed with the perfusate, the dialysate (multiBIC, Fresenius Medical Care) flow rate to and from the dialysis filter was set at a rate of $1,000-2,000 \mathrm{mlh}^{-1}$ to correct electrolytes and $\mathrm{pH}$ before connection of the liver to the perfusion system. The liver was connected to the system when sodium was in physiologic range and $\mathrm{pH}$ was around 7.2. Bicarbonates (sodium hydrogen carbonate $8.4 \%$, B. Braun Melsungen) were also used to correct $\mathrm{pH}$ at dialysis start if $\mathrm{pH}$ was below 7.0. During liver perfusion, the dialysate inflow rate to the dialysis filter was typically set to $200 \mathrm{mlh}^{-1}$. Dialysis outflow (leaving dialysis filter) rate was adjusted automatically by the control system depending on the measured hematocrit level. All the components of the perfusate are summarized in Supplementary Table 2. 
Pig livers. The experiments were performed in accordance with the Swiss Animal Protection Law and Ordinance. Female land race pigs (around $90 \mathrm{~kg}$ ) were obtained 1 week before surgery to allow acclimatization. The pigs were fasted overnight before the experiment with free access to water. The pigs were sedated with ketamine (Ketasol-100, $15 \mathrm{mg} \mathrm{kg}^{-1}$ ), azaperon (Stresnil, $2 \mathrm{mg} \mathrm{kg}^{-1}$ ) and atropinsulfate (Atropin 1\%, $0.05 \mathrm{mg} \mathrm{kg}^{-1}$ ). General anesthesia was maintained with isoflurane (Attane, 1-2\%) and propofol (Propofol Lipuro 1\%, 3-5 $\mathrm{mg} \mathrm{kg}^{-1} \mathrm{~h}^{-1}$ ). Laparotomy was performed under general anesthesia. The hepatic artery and portal vein were isolated for flow measurement with VeriQ (Medistim Germany $\mathrm{GmbH}$ ) according to the manufacturer's recommendation. Flow in the hepatic artery was measured distal to the splenic artery and after ligating gastric branches. Physiologic in vivo vascular flow rates in pigs for the hepatic artery and portal vein, measured at the time of organ procurement, were $0.25 \pm 0.071 \mathrm{~min}^{-1}(n=5)$ and $1.0 \pm 0.21 \mathrm{~min}^{-1}(n=5)$, respectively. These values served therefore as a reference for the ex vivo setting. Heparin $\left(200 \mathrm{IU} \mathrm{kg}^{-1}\right)$ was administered and the aorta was cannulated. Pig livers and blood were procured in standardized fashion ${ }^{22}$. Livers were connected to the perfusion machine after back-table preparation of the vessels (hepatic artery, portal vein, vena cava and bile duct). The circuit was primed with autologous, leukocyte-depleted pig blood (around 2.71) with addition of albumin $(200 \mathrm{ml}, 20 \%$ solvent $)$, bicarbonates $(20 \mathrm{ml})$, calcium gluconate $(10 \mathrm{ml})$, piperacillinum-tazobactamum $(2.2 \mathrm{~g})$ and methylprednisolon $(500 \mathrm{mg})$. The perfusion technology was developed in a step-by-step approach with the following stages as reported in the results section: glucose metabolism; minimizing hemolysis; electrolyte control; physiologic venous oxygen saturation in the portal vein; and liver movement. Pig livers were transplanted after ex vivo perfusion according to a previously established protocol ${ }^{22}$.

Sampling. Blood samples were taken before the start of perfusion, every $15 \mathrm{~min}$ during the first hours of perfusion and hourly for the next $5 \mathrm{~h}$. Thereafter, blood sampling was performed daily. Tissue samples (liver biopsies) were collected before the start of perfusion, after 3 and $6 \mathrm{~h}$ of perfusion and afterward on a daily basis. Bile was sampled daily.

Measurements. Perfusate. Blood-gas analysis from arterial (hepatic artery), portal (portal vein) and caval (vena cava) lines was performed using the Radiometer ABL90 FLEX. The blood-gas analysis data were used to calibrate the glucose sensor and the online sensors of the Terumo, CDI 500 device. A Piccolo Xpress analyzer (Abaxis) was used for the quantitative determination of BUN, albumin, alanine aminotransferase (ALT), AST, total bilirubin, gamma glutamyltransferase and alkaline phosphatase using the Piccolo general chemistry 13 panel (Abaxis). Ammonia levels were determined with the PocketChem BA (PA-4140, Arkray). Factor $\mathrm{V}$ was measured by the clinical laboratory of the University Hospital Zurich

Plasma from perfusate. Plasma samples were obtained from the blood-based perfusate by centrifugation using standard protocol and snap frozen in liquid nitrogen for further analysis (long-term preservation at $-80^{\circ} \mathrm{C}$ ). Free hemoglobin was measured spectrometrically assay using Drabkin solution (D5941, Sigma). The cytokine levels in the plasma were analyzed using LEGENDplex Human Inflammation Panel (740118, Biolegend). Fifty microliters of plasma were assayed following the manufacturer's instruction. In human livers 7 to 10, the upper measurable limit of the analytical method was taken for statistical analysis, because this value was exceeded. 8-Oxo-2'-deoxyguanosine (KA0444, Abnova) for DNA damage and cytochrome C (CSB-EL006328PI, Cusabio) for mitochondrial injury were measured with enzyme-linked immunosorbent assay according to the manufacturer's recommendation.

Tissue. Tissue biopsies were taken at different time points and positions before and during perfusion using standard protocols, and either stored in formalin for histological analysis or snap frozen in liquid nitrogen and stored at $-80^{\circ} \mathrm{C}$ for further analysis.

Histology. The following staining procedures were performed on random $3-\mu \mathrm{m}$ thick paraffin-embedded liver sections: hematoxylin and eosin, cleaved caspase 3 (9661S, Cell Signaling) staining as an apoptosis marker, von Willebrand Factor (IR527, DAKO) for endothelial cell activation, Ki-67 staining as a proliferation marker in bile ducts (IR626, DAKO), pH3 staining as a mitosis marker (M-06570-3KL, Milipore), CD68 staining for liver macrophages (M0876, DAKO) and cytokeratin 7 staining for bile ducts (DAKO). PAS staining was performed for qualitative analysis of glycogen content.

Frozen tissue. Total RNA was extracted from $10 \mathrm{mg}$ of liver tissue using RNeasy Mini kit (Qiagen) including a DNAse I digestion step (RNase-free DNase set, Quiagen) on column. The purified RNA was transcribed to cDNA using the qScript cDNA SuperMix reagent (VWR). TaqMan gene-expression assays and an 18S rRNA internal control (TaqMan ribosomal RNA control reagents) were from PE Applied Biosystems. Sequence amplification and data analysis were performed on the ABI Prism 7500 Sequence Detector system (PE Applied Biosystems). Taqman gene-expression assays used were Ss03384604_ul for porcine IL6, Ss03382372_ul for porcine IL10, Ss03392385_ml for porcine ICAM1, Hs00164932_m1 for human ICAM1 and 4308329 for 18S rRNA. The expression values were normalized to the sample at the perfusion start. Intrahepatic glycogen content was determined using the Glycogen Assay kit (MAK016, Sigma) following the manufacturer's instructions. Total protein was extracted from tissue $(10 \mathrm{mg})$ in RIPA buffer using the standard protocol. Protein quantification and immunoblots were performed using Biorad protocols and systems, and the antibodies used were phospho-Akt (S472) (9271S, Cell Signaling), $\alpha$-tubulin (2125S, Cell Signaling), phospho-GSK3 $\beta$ (S9) (5558S, Cell Signaling) and GSK3 $\beta$ (12456S, Cell Signaling). Mitochondrial function was analyzed by liver ATP content using ENLITEN rLuciferase/Luciferin Reagent (FF2021, Promega). In brief, the frozen tissue $(10 \mathrm{mg})$ was homogenized in $1.0 \mathrm{ml}$ of ice-cold TBS buffer and deproteinized using a TCA kit (ab204708, Abcam). One hundred microliters of diluted supernatant were assayed by addition of $50 \mu \mathrm{l}$ of ATP monitoring reagent. Luminiscence kinetics of samples and ATP standards (Sigma) were measured using a Citation 3 imaging reader (Biotek). ATP concentrations were extrapolated from the reading of the kinetic showing better regression of the calibration curve and normalized with the protein content in the samples before deproteinization.

Bile. DRI-CHEM 4000i (Fujifilm) was used for quantitative determination of total bilirubin. Bile glucose was measured using a Radiometer ABL90 FLEX device.

Statistics. Data collection and graphs were performed with Matlab R2017a (MathWorks). The data from different experiments were bundled at the same time points. Data points were bundled to a set when at least two data points of individual experiments were available. Then, the mean and s.d. of the bundled data points was calculated. Owing to logistical reasons, the daily sampling of the various experiments could not be conducted at exactly the same times, therefore they were also bundled over a time period of a $\pm 9 \mathrm{~h}$. For comparison of two groups, two-tailed Student's $t$ test was used. Data were reported as mean \pm s.d. $P<0.05$ was considered as significant. Exact sample size $(n)$ for each experimental group and units of measurement were provided in the text and figure captions. No assumptions or corrections were applied in statistical analysis. Further details on statistics are available online in the Reporting Summary.

Reporting Summary. Further information on research design is available in the Nature Research Reporting Summary linked to this article.

\section{Data availability}

Data in the manuscript will be made available upon reasonable request to the corresponding author.

\section{Acknowledgements}

We would like thank all the collaborators from the University Hospital Zurich for their expertise and substantial contributions in the development of the long-term perfusion technology: M. Halbe, R. Lenherr, B. Krueger, S. Segerer, N. Mueller, R. A. Schuepbach, S. Klinzing, B. Stieger, J.-D. Studt, K. Koch, M. Huellner, M. A. Schneider, M. Lipiski, M. Sauer, M. Canic, R. Schüpbach and M. Schlaepfer. We also thank F. Immer, B. Burg, D. Goslings, V. Figueiredo, B. Humar, M. Duskabilova, M. Tibbitt, E. Gygax, L. Mancina, A. Fleischli, C. Studer, V. T. Nguyen, S. Huber and A. Gupta. Special thanks go to Swisstransplant, the Swiss Donor Care Association and all of the organ donors and their families for their generous support, which enables research with human organs. The study was funded by a grant from Wyss Zurich, Helmut Horten Foundation, PROMEDICA Foundation and the Liver and Gastrointestinal foundation (LGID). We gratefully acknowledge this financial support. A.W. was supported by the Swiss National Science Foundation (320030_182764/1).

\section{Author contributions}

D.E., D.B., L.B.B., M.H., M.J.S., X.M., M.M., P.D., P.R.v.R. and P.-A.C. designed the perfusion machine and established the perfusion protocol, performed the perfusion experiments, generated and interpreted the data and wrote the manuscript. C.H. generated the data. C.O. designed the perfusion machine. R.G. established the perfusion protocol and interpreted the data. A.W. generated data and edited the manuscript.

\section{Competing interests}

ETH (Swiss Federal Institute of Technology in Zurich) and the University of Zurich (D.E., D.B., L.B.B., M.H., M.J.S., X.M., P.D., R. G., P.R.v.R. and P.-A.C.) have applied for a patent on this new perfusion technology (PCT/EP2019/051252).

\section{Additional information}

Supplementary information is available for this paper at https://doi.org/10.1038/ s41587-019-0374-X.

Correspondence and requests for materials should be addressed to P.-A.C.

Reprints and permissions information is available at www.nature.com/reprints. 


\section{Reporting Summary}

Nature Research wishes to improve the reproducibility of the work that we publish. This form provides structure for consistency and transparency in reporting. For further information on Nature Research policies, see Authors \& Referees and the Editorial Policy Checklist.

\section{Statistics}

For all statistical analyses, confirm that the following items are present in the figure legend, table legend, main text, or Methods section.

$\mathrm{n} / \mathrm{a}$ Confirmed

$\bigotimes$ The exact sample size $(n)$ for each experimental group/condition, given as a discrete number and unit of measurement

$\square$ A statement on whether measurements were taken from distinct samples or whether the same sample was measured repeatedly

The statistical test(s) used AND whether they are one- or two-sided

Only common tests should be described solely by name; describe more complex techniques in the Methods section.

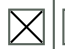
A description of all covariates tested

$\square$ \ A description of any assumptions or corrections, such as tests of normality and adjustment for multiple comparisons

$\square{ }^{A}$ A full description of the statistical parameters including central tendency (e.g. means) or other basic estimates (e.g. regression coefficient) AND variation (e.g. standard deviation) or associated estimates of uncertainty (e.g. confidence intervals) For null hypothesis testing, the test statistic (e.g. $F, t, r)$ with confidence intervals, effect sizes, degrees of freedom and $P$ value noted Give $P$ values as exact values whenever suitable.

Х $\square$ For Bayesian analysis, information on the choice of priors and Markov chain Monte Carlo settings

Х $\square$ For hierarchical and complex designs, identification of the appropriate level for tests and full reporting of outcomes

$\bigotimes \square$ Estimates of effect sizes (e.g. Cohen's $d$, Pearson's $r$ ), indicating how they were calculated Our web collection on statistics for biologists contains articles on many of the points above.

\section{Software and code}

\section{Policy information about availability of computer code}

\section{Data collection Matlab (MathWorks, USA)}

Data analysis Matlab (MathWorks, USA)

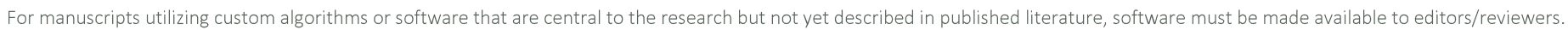
We strongly encourage code deposition in a community repository (e.g. GitHub). See the Nature Research guidelines for submitting code \& software for further information.

\section{Data}

Policy information about availability of data

All manuscripts must include a data availability statement. This statement should provide the following information, where applicable:

- Accession codes, unique identifiers, or web links for publicly available datasets

- A list of figures that have associated raw data

- A description of any restrictions on data availability

Request for data used in the manuscript should be addressed to corresponding author.

\section{Field-specific reporting}

Please select the one below that is the best fit for your research. If you are not sure, read the appropriate sections before making your selection.
\ Life sciences
Behavioural \& social sciences
Ecological, evolutionary \& environmental sciences

For a reference copy of the document with all sections, see nature.com/documents/nr-reporting-summary-flat.pdf 


\section{Life sciences study design}

All studies must disclose on these points even when the disclosure is negative.

Sample size No sample size calculation was performed in this study.

Data exclusions No data were excluded from the analysis

Replication The current liver perfusion technology was the results of a 3-year intensive development with preclinical experiments performed in swine livers with reproducible results. The merit of perfusion technology was tested finally with ten injured human livers.

Randomization Randomization is not relevant in this study with 10 injured human livers declined for transplantation and pig livers demonstrate feasibility of long term perfusion.

Blinding Authors were not blinded to data collection and analysis, since the measured results were required to maintain the liver perfusion and interpretation of liver performance.

\section{Reporting for specific materials, systems and methods}

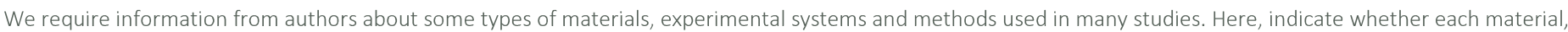

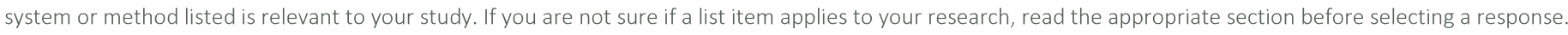

\begin{tabular}{|c|c|}
\hline $\mathrm{n} / \mathrm{a}$ & Involved in the study \\
\hline & $\bigotimes$ Antibodies \\
\hline Х & Eukaryotic cell lines \\
\hline$\bigotimes$ & $\square$ Palaeontology \\
\hline \begin{tabular}{|l|l} 
\\
\end{tabular} & $\bigotimes$ Animals and other organisms \\
\hline$\square$ & $\bigotimes$ Human research participants \\
\hline Х & $\square$ Clinical data \\
\hline
\end{tabular}

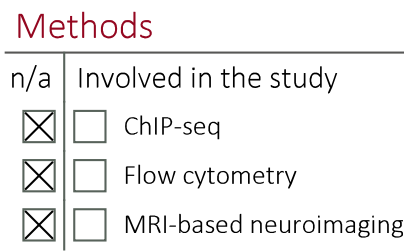

Antibodies

Antibodies used

Data provided in the manuscript

Validation

Data provided in the manuscript

\section{Animals and other organisms}

Policy information about studies involving animals; ARRIVE guidelines recommended for reporting animal research

Laboratory animals

Wild animals

Field-collected samples

Ethics oversight

\section{Female land race pigs}

The study did not involve wild animals

The study did not involve samples collected from the field.

The experiments were performed in accordance with the Swiss Animal Protection Law. The local animal walfare committe approved the study protocol.

Note that full information on the approval of the study protocol must also be provided in the manuscript.

\section{Human research participants}

Policy information about studies involving human research participants

Population characteristics

Recruitment

Ethics oversight
Human liver grafts declined for transplantation. The donor and grafts details were provided in Supplementary Table 1.

Ten human livers were obtained for research after being declined for transplantation nationwide, and the consent was obtained from the next of kin.

The local ethics committee of Canton of Zurich approved the study protocol (2017-000412). 
